\title{
On the formation of axial corner vortices during spin-up in a cylinder of square cross section
}

\author{
R. J. MUNRO1, R. E. HEWITT ${ }^{2}$, AND M. R. FOSTER \\ ${ }^{1}$ Faculty of Engineering, University of Nottingham, Nottingham, NG7 2RD, UK \\ ${ }^{2}$ School of Mathematics, University of Manchester, Manchester, M13 9PL, UK \\ ${ }^{3}$ Department of Mathematical Sciences, Rensselaer Polytechnic Institute, Troy, NY 12180, \\ USA
}

(Received 8 April 2015)

We present experimental and theoretical results for the adjustment of a fluid (homogeneous or linearly stratified), which is initially rotating as a solid body with angular frequency $\Omega-\Delta \Omega$, to a nonlinear increase $\Delta \Omega$ in the angular frequency of all bounding surfaces. The fluid is contained in a cylinder of square cross section, which is aligned centrally along the rotation axis, and we focus on the $O\left(R o^{-1} \Omega^{-1}\right)$ time scale, where $R o=\Delta \Omega / \Omega$ is the Rossby number. The flow development is shown to be dominated by unsteady separation of a viscous side wall layer, leading to an eruption of vorticity that becomes trapped in the four vertical corners of the container. The longer-time evolution on the standard "spin-up" time scale, $E^{-1 / 2} \Omega^{-1}$ (where $E$ is the associated Ekman number), has been described in detail for this geometry by Foster \& Munro (J. Fluid Mech., vol. 712, 2012, p. 7-40), but only for small changes in the container's rotation rate (i.e. $R o \ll 1$ ). In the linear case, for $R o \ll E^{1 / 2} \ll 1$, there is no side-wall separation. In the present investigation we focus on the fully nonlinear problem, $R o=O(1)$, for which the side wall viscous layers are Prandtl boundary layers and (somewhat unusually) periodic around the container's circumference. Some care is required in the corners of the container, but we show that the side wall boundary layer breaks down (separates) shortly after an impulsive change in rotation rate. These theoretical boundary layer results are compared to two-dimensional Navier-Stokes results, which capture the eruption of vorticity, and these are in turn compared to laboratory observations and data. The experiments show that when the Burger number, $S=(N / \Omega)^{2}$ (where $N$ is the buoyancy frequency), is relatively large - corresponding to a strongly stratified fluid - the flow remains (horizontally) two-dimensional on the $O\left(R o^{-1} \Omega^{-1}\right)$ time scale, and good quantitative predictions can be made by a two-dimensional theory. As $S$ was reduced in the experiments, three-dimensional effects were observed to become important in the core of each corner vortex, on this time scale, but only after the break down of the sidewall layers.

\section{Introduction}

The term spin-up is most commonly used to describe how a fluid adjusts from an initial, to a new state of solid-body rotation, due to an increase in the rotation speed of the fluid's confining boundaries. Many previous studies have focused on the linear spin-up of a homogeneous fluid in a closed circular cylinder. By 'linear' we mean the Rossby number $R o=\Delta \Omega / \Omega$ is small, where $\Omega-\Delta \Omega$ and $\Omega$ are the initial and new angular frequencies of the cylinder, respectively. In this case we know the flow is axisymmetric and the fluid 
is spun up by a meridional-plane secondary circulation in the fluid's interior, driven by the Ekman boundary layers at the cylinder's base and lid (Greenspan \& Howard 1963; Benton \& Clark 1974; Duck \& Foster 2001). This secondary circulation stretches the background vortex and gradually draws fluid radially inwards, which by conservation of angular momentum must acquire greater angular velocity. As a result, the fluid adjusts from the old to the new rotation speed exponentially, on the time scale $E^{-1 / 2} \Omega^{-1}$, where $E=\nu / \Omega L^{2}$ is the Ekman number, $\nu$ is the fluid's kinematic viscosity and $L$ is a characteristic length scale, such as the fluid's depth or a measure of the container size. In the vast majority of practical applications $E \ll 1$, and so the spin-up time scale is large compared to the formation time of the Ekman layers, which is of order $\Omega^{-1}$, but much smaller than the viscous diffusion time scale, $E^{-1} \Omega^{-1}$. The spin-up time scale is derived theoretically for linear spin-up (Greenspan \& Howard 1963). However, it has been shown to also apply to the nonlinear regime - where $R o$ is not small - and to the limiting case of spin-up from rest, $R o=1$ (Weidman 1976).

The presence of a stable density stratification further complicates the spin-up process. This problem was studied by Walin (1969), who described the linear spin-up $(R o \ll 1)$ of a linearly stratified fluid in a closed circular cylinder. In this case, the stable density field inhibits vertical motion. In particular, Walin (1969) showed that the radial Ekman-layer flux, on reaching the perimeter of the base (and lid), cannot be transported vertically within sidewall shear layers - as is the case in homogeneous spin-up (Stewartson 1957) but instead erupts into the inviscid interior region. As a result, there is no meridional circulation throughout the fluid's interior. Instead, the spin-up process only penetrates to a height (depth) of order $S^{-1 / 2} L$, where $S=(N / \Omega)^{2}$ is the Burger number and $N$ the fluid's buoyancy frequency. Consequently, the limiting steady-state on the spin-up time scale is a spatially non-uniform rotation, with the degree of non-uniformity governed by the magnitude of $S$. The final spun-up state is approached only on the much longer viscous time scale, $E^{-1} \Omega^{-1}$. More recent investigations of analogous nonlinear problems can be found in Thomas \& Rhines (2002), Smirnov et al. (2005) and Munro et al. (2010).

The stratified spin-up process is further complicated by the presence of boundaries that are sloped at an angle to the axis of rotation, in which case the near boundary flow is a buoyancy-inhibited Ekman layer. A linearised description of such flows was presented by MacCready \& Rhines (1991), whilst a nonlinear description of the nearboundary flow was presented by Duck et al. (1997) in the context of a conical container. The nonlinear theory shows that the near-wall boundary layer typically thickens with time, although at sufficiently low levels of density stratification steady state flows or boundary layer breakdown is possible. Experimental comparisons for such buoyancyinhibited layers were presented by Hewitt et al. (1999), again for conical containers and linear stratification. Even for the simpler case of two-layer stratification, if the density interface intersects sloping boundaries an algebraic (in time) linear spin-up response can be achieved, as described by Hewitt et al. (2001).

Van Heijst (1989) was the first to study spin-up in non-axisymmetric containers with bounding walls that were either perpendicular or parallel to the axis of rotation. Laboratory experiments were used to observe the spin-up from rest $\left(R_{o}=1\right)$ of a homogeneous fluid (with a free surface) in a variety of tank geometries, including a semi-circular cylinder and an annular cylinder with a radial barrier. The follow-up studies by van Heijst et al. (1990) and van de Konijnenberg \& van Heijst (1997) used containers with rectangular cross section, and both homogeneous and linearly stratified fluids. At early times, the motion relative to a coordinate frame that rotates with the container is a horizontal, anticyclonic flow, that completely fills the interior domain. A feature of such flows is the presence of horizontal pressure gradients along the container's sidewalls, which led to the 
conjecture that unsteady flow separation may occur. Shortly after the rotation starts, vorticity generated in the sidewall boundary layers is advected into the fluid's interior, forming cyclonic line vortices that interact with, and break-up, the initial starting flow. The background rotation eventually stabilizes the flow into an organised array of alternately cyclonic and anticyclonic vortices (or cells), whose number and size are determined by the container's geometry and horizontal aspect ratio. This flow pattern persists, but gradually decays on the $E^{-1 / 2} \Omega^{-1}$ time scale due to the drag associated with the Ekman layer at the base of each cell.

More recently, Foster \& Munro (2012) reported a theoretical and experimental study of the linear spin-up $(R o \ll 1)$ of a linearly stratified fluid in a closed cylinder with a square cross-section. Their experimental results showed that the detachment of the sidewall boundary layers results in the formation of an equally-sized cyclonic line vortex in each of the cylinder's vertical corners. The two-dimensional anticyclonic starting flow, which initially fills the flow domain, is deformed by the formation of the corner vortices but throughout remains dominant and centred about the cylinder's vertical axis. The weaker cyclonic corner vortices remain confined to the corner regions. This is in contrast to a rectangular domain (van Heijst et al. 1990), where the corner vortices form asymmetrically, with large vortices developing in the corner regions downstream of the two long sides, which grow to a size comparable with the container's width and then interact with the initial starting-flow cell.

Foster \& Munro (2012) derived an asymptotic result to describe how the starting flow in a square cylinder is spun-up on the time scale $E^{-1 / 2} \Omega^{-1}$. Their result accounted for two effects. Firstly, Ekman compatibility conditions were applied in combination with a condition for the singular Ekman-layer eruptions to account for how the interior flow is spun-up by the drag associated with the Ekman layers at the cylinder's base and lid. Secondly, they showed that on the spin-up time scale the boundary layers for the horizontal velocity components tangential to each sidewall take the form of inwardly growing Rayleigh layers. The composite solution combining these elements was shown to exhibit an excellent level of agreement with experimental data. Notably, this asymptotic result - valid for $R o \ll E^{1 / 2}$ - does not account for the corner-cell formation. However, Foster \& Munro (2012) showed that for times of order $R o^{-1} \Omega^{-1}$ the sidewall shear layers are conventional Prandtl boundary-layers, which led them to the conjecture that the corner cells form on this time scale, and are the result of a finite-time singularity in the (nonlinear) Prandtl equations in the region of adverse pressure-gradient upstream of each corner. Although no formal analysis of this singularity was attempted, evidence from their experimental observations did support this conjecture, and confirmed that the formation of the corner cells was complete for times of order $R o^{-1} \Omega^{-1}$.

Here, we also consider spin-up in a square cylinder. Our attention is focused on reporting, for the first time, a detailed experimental and theoretical description of how axial vortices form in the cylinder's vertical corner regions on the $R o^{-1} \Omega^{-1}$ time scale, and on how their formation is affected by density stratification of the fluid. The sidewall boundary-layer equations derived by Foster \& Munro (2012) are reformulated and analysed to investigate the issue of unsteady separation; the theoretical predictions and computations are compared with experimental observations. Here we do not consider the longer spin-up time scale, and so the Ekman number has less relevance. Instead, the theoretical analysis reported here shows that the key parameter for the sidewall boundary layers, on the $R o^{-1} \Omega^{-1}$ time scale, is the Reynolds number, $R e=\Delta \Omega L^{2} / \nu=R o / E$, where $L$ denotes the cylinder's width. Foster \& Munro (2012) studied stratified, linear spin-up for values of $R o$ between 0.01 and 0.1 and $S$ in the range 0.2 to 26, with $R e$ between 970 and 2800. The corner regions observed in their experiments had a simple 
structure, consisting of a single, cyclonic line vortex extending over the fluid's depth. In this paper we consider the case $R e=\mathcal{O}\left(10^{4}\right)$, with $S$ ranging between 0 to 100 . We will show that this case gives rise to the formation of multiple line vortices in each corner region, both cyclonic and anticyclonic. Van de Konijnenberg (1997) reported two experiments on spin-up from rest in a square cylinder with $R e=\mathcal{O}\left(10^{5}\right)$, and in both cases the corner vortices were observed to become fully turbulent shortly after formation. Here, attention has been restricted to $R e \lesssim 40000$, which for our configuration meant that the vortices that form in the corner regions remained laminar. The theoretical work reported here applies to the general case $R o \leqslant 1$. For simplicity, however, we present experimental data only for the case $R o=1$ (spin-up from rest). Experiments were performed for $R o<1$, but no significant differences were observed.

The paper is structured as follows. In section 2 we describe the experimental set-up and observations. In section 3 we present the theoretical analysis of the flow, describing the evolution of the unsteady side wall boundary layers. Section 3 also presents the results of two-dimensional Navier-Stokes computations, which are used to reinforce the asymptotic analysis over time scales, of $\mathcal{O}\left(\Omega^{-1}\right)$, which remain short compared to the time taken for spin-up of the contained fluid. Some final remarks and conclusions are given in section 4 . Some theoretical justification for the two-dimensionality assumption made in the computations is given in an Appendix.

\section{Experiments}

\subsection{Apparatus and flow visualisation}

The experimental configuration is shown in figure 1. Each experiment was performed in a transparent square cylinder (of width $L=38 \mathrm{~cm}$, height $H=51 \mathrm{~cm}$ ) mounted on a variable-speed turntable with its vertical axis aligned with the rotation axis of the table (see figure 1). The cylinder was filled with either (i) a uniform-density salt-water solution, or (ii) a linearly stratified salt-water solution with a buoyancy frequency $(N)$ of 0.30 or $0.83 \mathrm{~Hz}$. In both cases, the salt used was $\mathrm{NaCl}$. The linear density stratification was set-up, and the corresponding density gradient measured, using conventional techniques similar to those described by Economidou \& Hunt (2009). Once filled, the cylinder was sealed with a transparent rigid lid, which was fitted to completely displace the fluid's free surface, so that no air pockets were trapped on the lid's underside. The sealed cylinder was left to stand for several hours to allow the fluid to reach a quiescent state. The experiment was then initiated (at time $t^{*}=0$ ) by smoothly increasing the table's rotation rate, from rest, to the final angular frequency $\Omega$, which was thereafter held fixed for the experiment's duration. The relative variability in $\Omega$ was always small and less than $3 \%$. In all cases the ramp time (i.e. the period over which the table's rotation rate was increased) was between 3 and 4 sec, depending on the magnitude of $\Omega$.

The flow (relative to a frame that rotates with the container) was visualized and measured using particle streaklines and PIV (Dalziel 2006). To facilitate the use of these techniques, small tracer particles were added to the fluid once the cylinder had been filled (and before fitting the lid). When added to the uniform-density fluid, the particles were made neutrally buoyant by dissolving sufficient salt into the water to match the mean density of the particles (i.e. $1.027 \mathrm{~g} / \mathrm{cm}^{3}$ ); the fluid was thoroughly stirred to ensure the particles were uniformly distributed. When added to the linearly stratified fluid, the particles were allowed to settle freely into suspension in a narrow band about their mean buoyancy level within the fluid's density stratification. In both cases, a thin (co-rotating) horizontal light sheet was directed through the cylinder's sidewall, at a height $z_{0}$ above 


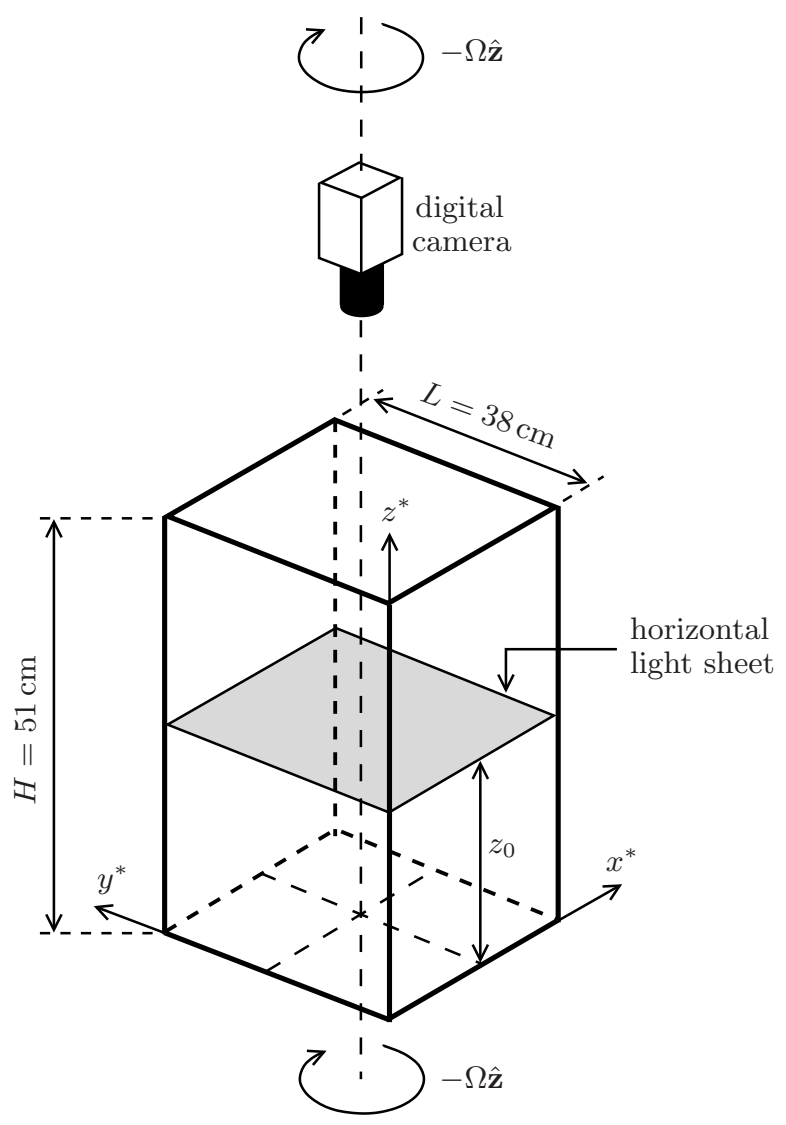

FIGURE 1. A sketch of the basic experiment set-up. The co-rotating coordinate system $\left(x^{*}, y^{*}, z^{*}\right)$ is also shown ( $\hat{\mathbf{z}}$ denotes the unit $z$-direction). The cylinder's rotation was in the clockwise direction.

the cylinder's base (see figure 1), to illuminate the suspended particles located within this horizontal plane. (For the linearly stratified fluid, $z_{0}$ corresponded to the neutral buoyancy level of the particles.) Experiments were performed with $z_{0} / H$ in the range 0.2 to 0.6 . The horizontal light sheet was produced using two Xenon arc lamps mounted inside a light box, which was designed to only emit light through a narrow, horizontal slit in its front panel. The light box was mounted on the turntable with the light sheet positioned at the desired height $z_{0}$.

Following the onset of the table's rotation (at time $t^{*}=0$ ), the relative motion of the illuminated particles was captured using a co-rotating digital video camera mounted on the turntable and positioned to view vertically down into the cylinder's interior (see figure 1 ). The images were recorded at a frame rate of $15 \mathrm{~Hz}$, and were processed at the end of each experiment relative to the co-rotating coordinates, $\left(x^{*}, y^{*}, z^{*}\right)$, which are shown in figure 1. Firstly, streakline images of the tracer particles were generated by taking time exposures of consecutive images (typically over a 2 or $3 \mathrm{sec}$ duration), examples of which are shown in figure 2. This exposure time is small compared to the time it takes for the cylinder to complete one revolution, and so the streaklines provide useful estimates of the instantaneous streamlines. Secondly, measurements of the horizontal velocity components $\left(u^{*}, v^{*}\right)$, in the horizontal plane $z^{*}=z_{0}$, were obtained by applying a standard PIV algorithm (Dalziel 2006) to consecutive images. 


$\begin{array}{clccc}\text { Label } & \Omega[\mathrm{rad} / \mathrm{s}] & S & R e & z_{0} / H \\ \mathrm{~A} & 0.083 & 100 & 12000 & 0.26 \\ \mathrm{~B} & 0.13 & 44 & 18000 & 0.26 \\ \mathrm{C} & 0.19 & 20 & 27000 & 0.26 \\ \mathrm{D} & 0.070 & 18 & 10000 & 0.31 \\ \mathrm{E} & 0.11 & 7.6 & 16000 & 0.31 \\ \mathrm{~F} & 0.24 & 1.6 & 34000 & 0.43 \\ \mathrm{G} & 0.28 & 0 & 41000 & 0.51 \\ \mathrm{H} & 0.18 & 0 & 27000 & 0.46 \\ \mathrm{I} & 0.14 & 0 & 20000 & 0.29 \\ \mathrm{~J} & 0.091 & 0 & 13000 & 0.29 \\ \mathrm{~K} & 0.055 & 0 & 8000 & 0.60\end{array}$

TABle 1. A list of the important experimental parameters. In all cases, $R o=1$.

All experiments reported here were performed from an initial state of rest $\left(R_{o}=1\right)$, with Re ranging from 8000 to 41000 , and with the Burger number $S=(N / \Omega)^{2}$ between 0 and 100, where $S=0$ corresponds to uniform-density fluid (i.e. $N=0$ ). In table 1 , the key parameters are listed for each experiment, which are henceforth labelled A to K. Throughout, $E^{1 / 2}=\mathcal{O}\left(10^{-2}\right)$ or less, so the standard spin-up time scale $\left(E^{-1 / 2} \Omega^{-1}\right)$ was always large compared to the time scale associated with the formation of the corner vortices $\left(R o^{-1} \Omega^{-1}\right)$. For the case considered here of salt $(\mathrm{NaCl})$ dissolved in water, the Schmidt number was $S c \approx 670$ (Munro et al. 2010). That is, the molecular diffusion of salinity in the stratified-fluid experiments was not significant. Finally, we note that the direction of the turntable's rotation was clockwise in all experiments, so we denote the final angular frequency vector of the cylinder by $\Omega=-\Omega \hat{\mathbf{z}}$ (with $\Omega>0$ ), where $\hat{\mathbf{z}}$ is the unit vector in the $z$-direction.

\subsection{Observations}

Here we introduce the dimensionless time, coordinates and corresponding velocity components defined as

$$
t=\Omega t^{*}, \quad(x, y, z)=L^{-1}\left(x^{*}, y^{*}, z^{*}\right), \quad(u, v, w)=(L \Omega)^{-1}\left(u^{*}, v^{*}, w^{*}\right) .
$$

The streakline images in figure $2(R o=1, R e=12000, S=100)$ show the key stages observed as the vortices form in the vertical-corner regions of the cylinder. We will now focus attention on this formation period, which corresponds (approximately) to the first rotation period of the cylinder.

A more detailed discussion of the flow response will be presented in section 2.2.2, but we begin with an initial presentation of the generic features of the flow, in the context of spin up from rest. Once the cylinder is set in rotation, the starting flow is immediately established. The starting flow is inviscid and two-dimensional, and takes the form of an anticyclonic rotation that entirely fills the cylinder's interior, with closedpath streamlines (figure $2 a$ ). Subsequent to this, the boundary layers that form along each sidewall appear to show a break down upstream of each corner region, where the 
(a)

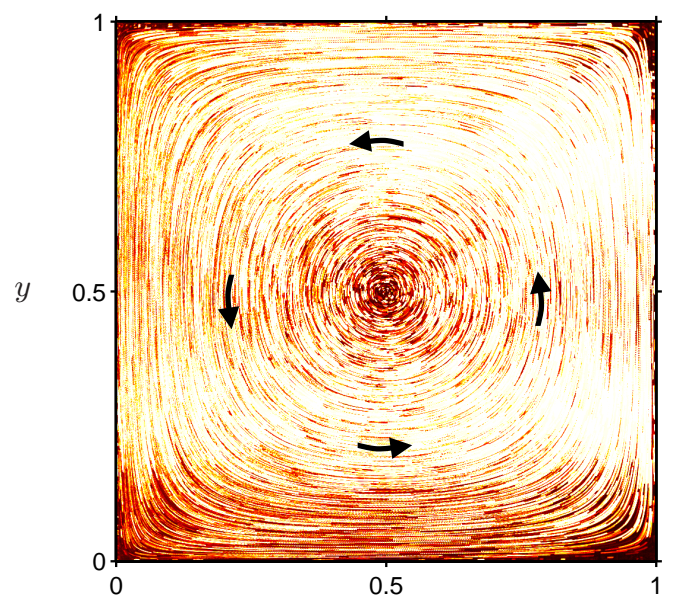

$(c)$

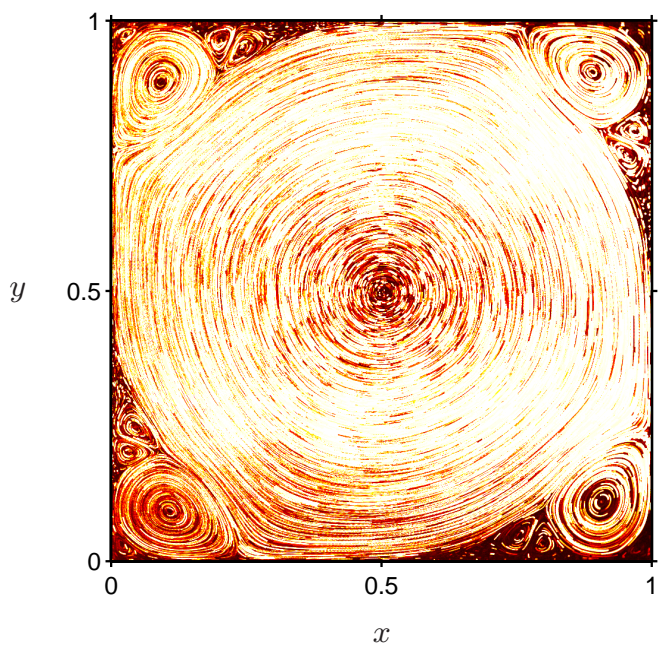

(b)

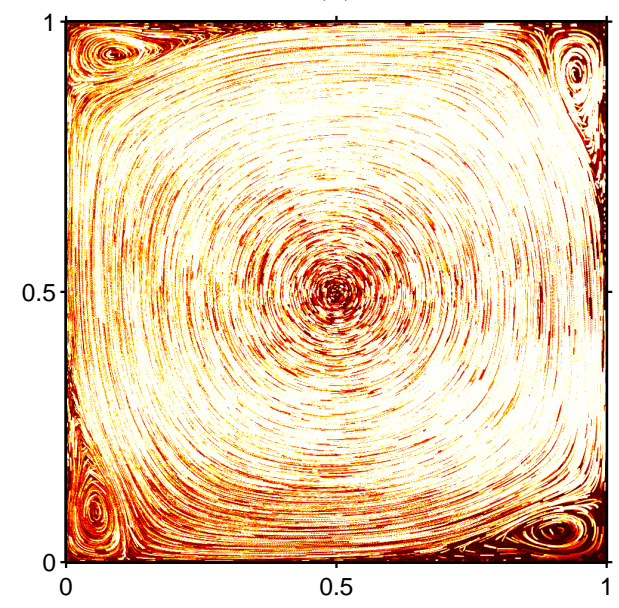

$(d)$

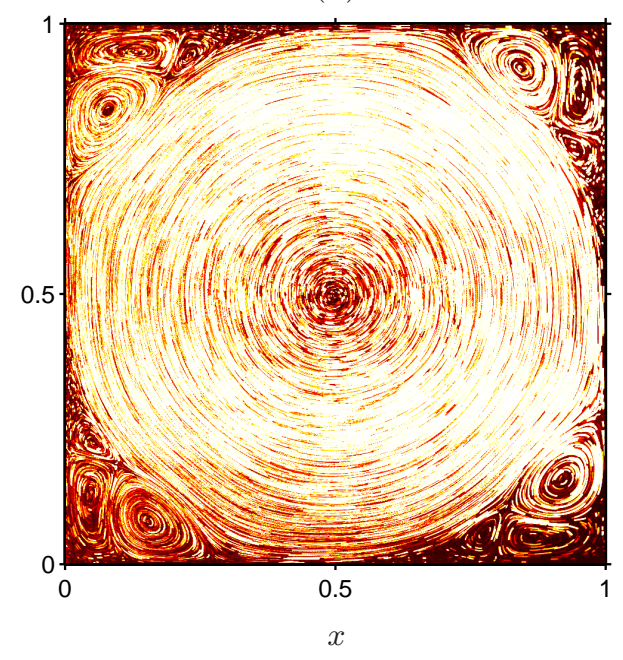

Figure 2. Streakline images showing key stages during the relative flow's evolution. The data shown are from experiment A $\left(R o=1, R e=12000, S=100, \Omega=0.083 \mathrm{rad} / \mathrm{s}, z_{0} / H=0.26\right)$. The exposure time for each image was $3 \mathrm{sec}$, and the corresponding dimensionless times are $t=\Omega t^{*}=(a)$ 0.33, $(b)$ 1.8, $(c) 3.3$, and $(d)$ 7.8. (Each of these times corresponds to the middle of the exposure.) The cyclonic direction is clockwise, so the relative starting-flow in $(a)$ is in the anticlockwise direction, as indicated by the arrows. Note that, $t=2 \pi$ corresponds to the first rotation period of the cylinder.

sidewall pressure gradient is adverse; at such a "break down" the boundary layer is no longer a thin region attached to the sidewall. As a result, cyclonic vorticity is ejected from the sidewall boundary layers and advected by the interior flow, accumulating in the adjacent downstream corner region and forming a cyclonic axial vortex that extends over the cylinder's depth. A vortex forms simultaneously in each of the four corner regions of the cylinder cross section, such that the flow pattern is invariant under rotation by $\pi / 2$, as shown in figure $2(b)$. As these corner vortices develop, additional anticyclonic vortices are produced adjacent to the boundary, which interact with and deform the original corner vortices (see figure $2 c$ ), eventually leading to the formation of three alternately 
cyclonic and anticyclonic axial vortices in each of the four vertical corner regions of the cylinder (figures $2 d$ ).

The key stages outlined above were generally observed in all experiments reported here. The notable exceptions to this were the experiments performed at the higher range values of $R e$ (between 37000 and 40000); in these cases the corner regions were turbulent shortly after formation. We also highlight that the experiment shown in figure 2 corresponds to large Burger number (i.e. $S=100$ ). In this case the fluid's density stratification is sufficiently strong to inhibit vertical motions, and so the flow remains essentially twodimensional. This was not the case for experiments at intermediate and small $S$, and in particular at $S=0$, where vertical motion was observed in the corner regions shortly after their formation. Hence, we will now describe in detail (see section 2.2.2 below) the flow's evolution through the key stages outlined above, and how this evolution differs with $S$. To begin, however, we briefly summarize the key features of the early-time starting flow (shown in figure $2 a$ ), which although equivalent to the starting flow described previously by Foster \& Munro (2012) and van Heijst et al. (1990), the details are briefly repeated here as they will be needed later in section 3 in our analysis of the sidewall boundary layers.

\subsubsection{The inviscid, two-dimensional starting flow}

The cylinder starts rotating at $t=0$, giving rise to the starting flow (relative to a frame that rotates with the container) is shown in figure $2(a)$, which is an anticyclonic rotation entirely filling the cylinder's interior. At this early time the boundary layers along the lid, base and sidewalls are still forming and so have had no discernible effect on the interior flow. In particular, the Ekman suction is weak (i.e. $\mathcal{O}\left(t^{1 / 2} E^{1 / 2}\right)$ ) at early times and so there is effectively no vertical motion $(w=0)$. The starting flow is therefore largely inviscid, two-dimensional and conserves the total vorticity of the initial condition, which relative to the co-rotating reference frame is given dimensionally by $2 \Omega \hat{\mathbf{z}}$. Under these conditions, and following the approach used for other non-axisymmetric geometries (van Heijst 1989; van Heijst et al. 1990), the flow within the interior can be represented by a two-dimensional streamfunction, $\psi(x, y)$, related to the horizontal velocity components by $(u, v, 0)=-\hat{\mathbf{z}} \times \nabla \psi=\left(\psi_{y},-\psi_{x}, 0\right)$. Conservation of vorticity requires that $\psi$ satisfies

$$
\nabla^{2} \psi=-2,
$$

and with the boundary layers along each sidewall neglected, the relevant boundary conditions for $(2.2)$ are that $\psi=0$ at $x=0,1$ and $y=0,1$. The corresponding solution can be written as

$$
\psi=x(1-x)-\frac{8}{\pi^{3}} \sum_{n=1}^{\infty}\left\{\frac{\sinh [\pi(2 n-1) y]+\sinh [\pi(2 n-1)(1-y)]}{(2 n-1)^{3} \sinh [\pi(2 n-1)]}\right\} \sin [\pi(2 n-1) x] .
$$

Figure 3 shows a qualitative comparison between the streakline data in figure 2( $a$ ) with a sample of contours generated from (2.3), together with a quantitative comparison between measured and theoretical velocities (see caption for details).

\subsubsection{Formation of the corner regions}

The corner regions form during the first rotation period of the cylinder, and the key stages of the flow's development are shown in figure 2. During this period, the flow is the same in the four corner regions, and so we now describe this formation sequence using the streakline images in figure 4 , which show a close-up view of the quadrant $0 \leqslant x \leqslant 0.5$, 
(a)

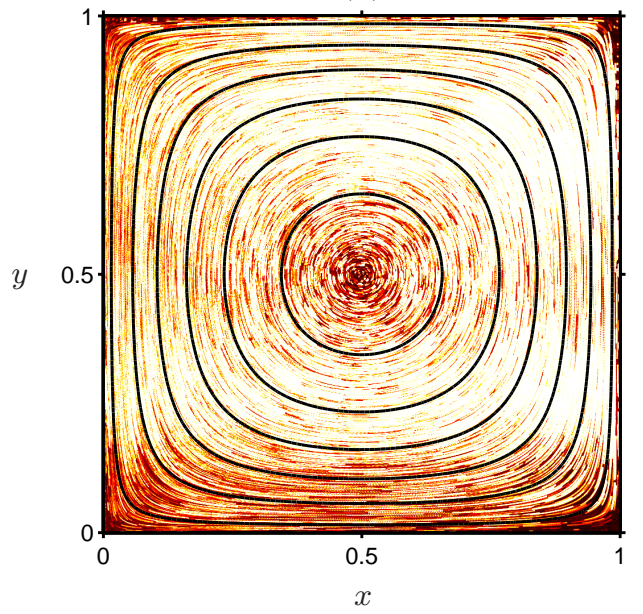

(b)

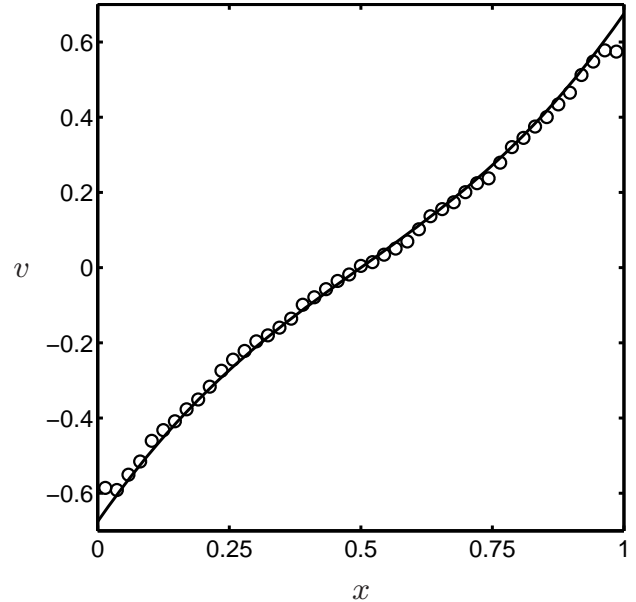

Figure 3. (a) The solid lines show contours of the streamfunction (2.3) superimposed on the streakline data shown previously in figure $2(a)$. (b) The solid line shows the velocity component $v=-\psi_{x}$, evaluated using (2.3) along the symmetry axis $y=1 / 2$, and compared with corresponding experimental data (o). The experimental parameters are $R o=1, R e=12000, S=100$, $\Omega=0.083 \mathrm{rad} / \mathrm{s}, z_{0} / H=0.26$ (experiment A) and the data are shown for $t=\Omega t^{*}=0.33$.

$0 \leqslant y \leqslant 0.5$, focussed on the corner at $(x, y)=(0,0)$. Note, the data in figures 2 and 4 are from experiment A $\left(R o=1, R e=12000, S=100, z_{0} / H=0.26\right)$.

Once the cylinder is set in rotation, the two-dimensional starting flow is quickly established. Shortly after this, as seen in figure 4(a) and 4(b), there is visual evidence to suggest that the boundary layer that has formed on the $(x=0)$ sidewall upstream of the corner is reversing, in the region where the tangential horizontal pressure gradient is adverse. Cyclonic vorticity generated in the boundary layer is advected into the corner, where it forms an axial vortex (figures $4 a$ and $4 b$ ) which extends over the tank's depth. (We henceforth denote this cyclonic corner vortex as the "primary".) At the time shown in figure $4(a)$, the streamline at the perimeter of the starting flow has detached from the $x=0$ sidewall at $y \approx 0.25$, and reattached to the adjacent $y=0$ sidewall at $x \approx 0.05$. As more cyclonic vorticity is advected into the corner region, the primary vortex grows in cross-section and the detachment (reattachment) point moves upstream (downstream) along the sidewall (figures $4 b$ and $4 c$ ).

The continued development of the primary vortex results in the formation of two distinct "secondary" vortices. In figure 4(c), the first of these secondary vortices can be seen adjacent to the $x=0$ sidewall, with its axis at $y \approx 0.2$. The second, which forms shortly after, is situated in the region between the corner's apex and the primary vortex, and can be seen in figure $4(d)$. Like the primary, the axes of these secondary vortices extend over the cylinder's depth. As more anticyclonic vorticity is supplied to the secondary vortices, they grow in cross-section. Notably, the growth of the upstream secondary vortex (at $y \approx 0.2$ in figure $4 c$ ) deforms and then pinches the primary vortex, which eventually divides (figure $4 d$ ). Moreover, as the secondary vortices grow they subsequently merge to form a single anticyclonic vortex. The merging event is shown in figure 4(e), and the single merged anticyclonic vortex is shown in figure $4(f)$, occupying the region adjacent to the corner's apex, flanked either side by the two divided components of the (cyclonic) 
(a)

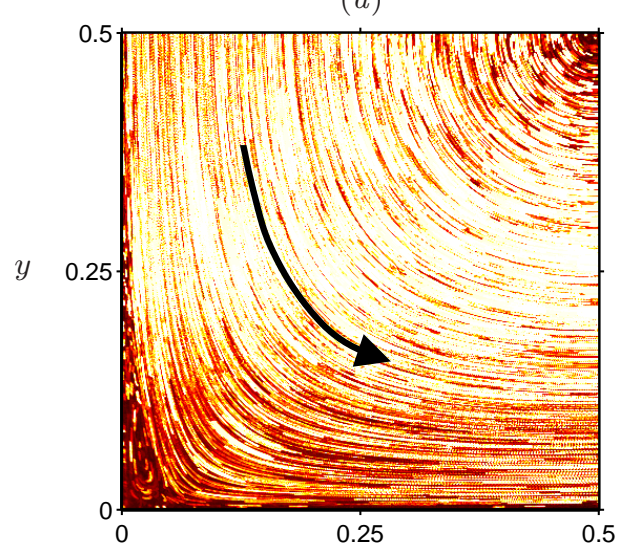

(c)

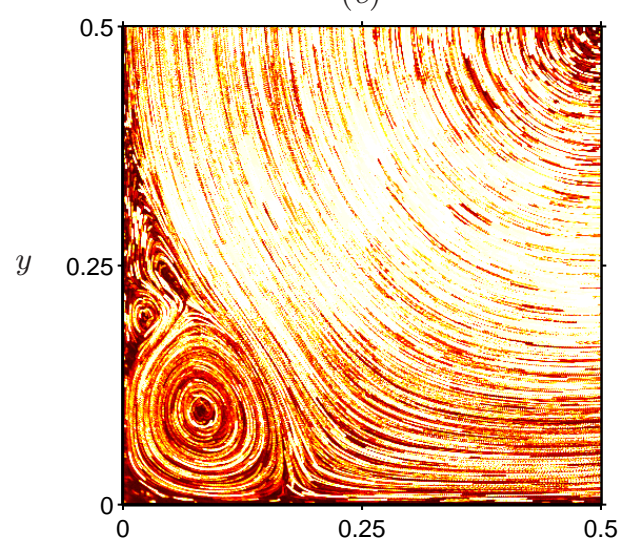

$(e)$

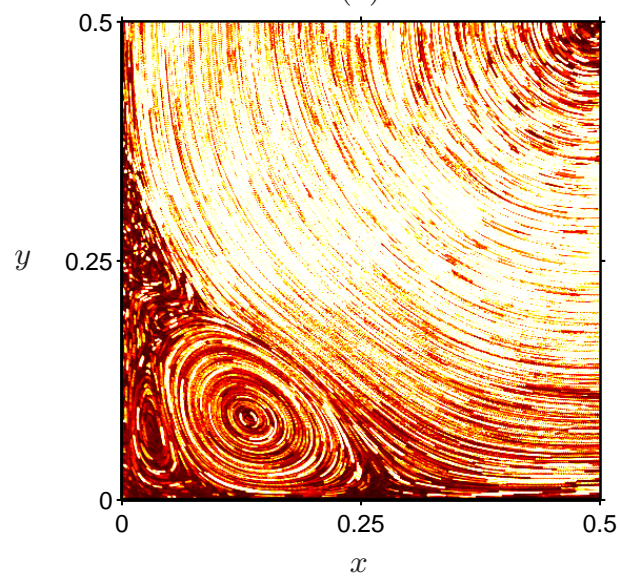

(b)

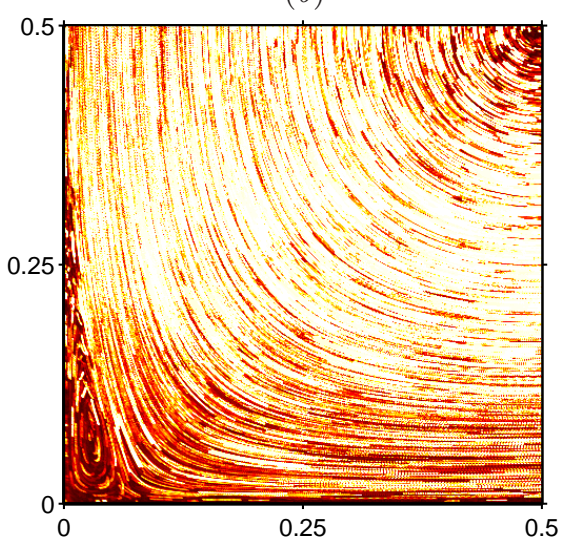

$(d)$

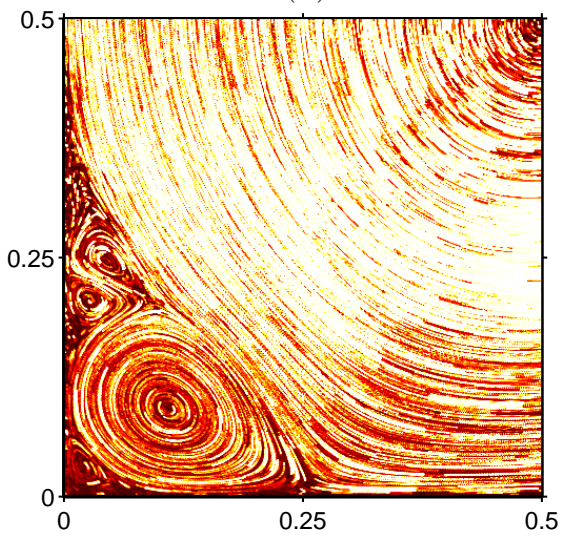

$(f)$

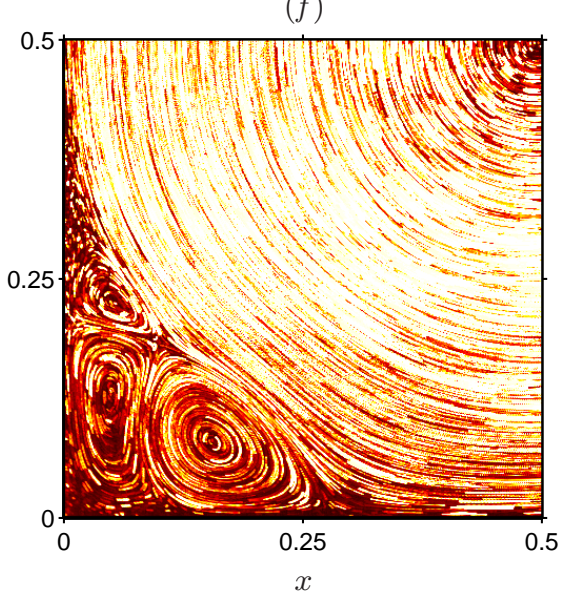

FiguRE 4. Experiment A: $R o=1, R e=12000, S=100, \Omega=0.083 \mathrm{rad} / \mathrm{s}, z_{0} / H=0.26$. Streakline images taken at dimensionless times $t=\Omega t^{*}=(a) 0.83,(b) 1.1,(c) 2.6,(d) 3.2$, (e) 6.6, $(f) 7.8$; note that $t=2 \pi$ corresponds to the first rotation period of the turntable. The exposure time for each image was $3 \mathrm{sec}$. The black arrow in $(a)$ has been included to show the direction of the anticyclonic interior flow. 
(a)

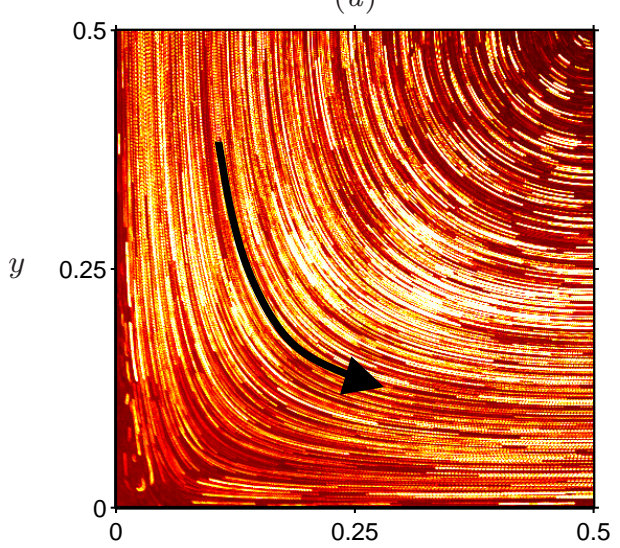

(c)

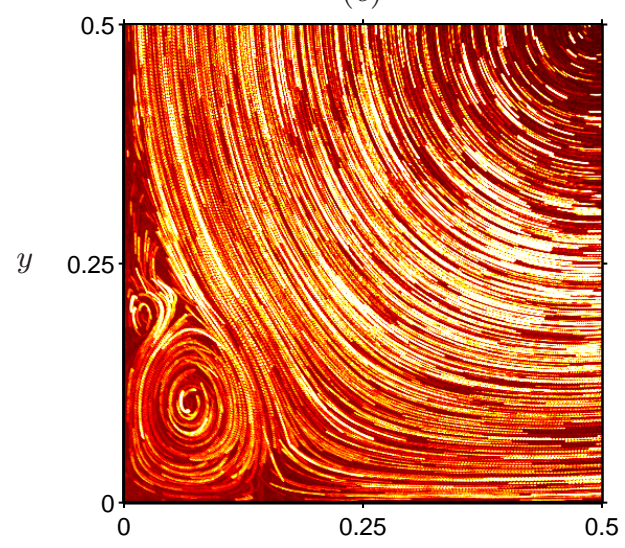

$(e)$

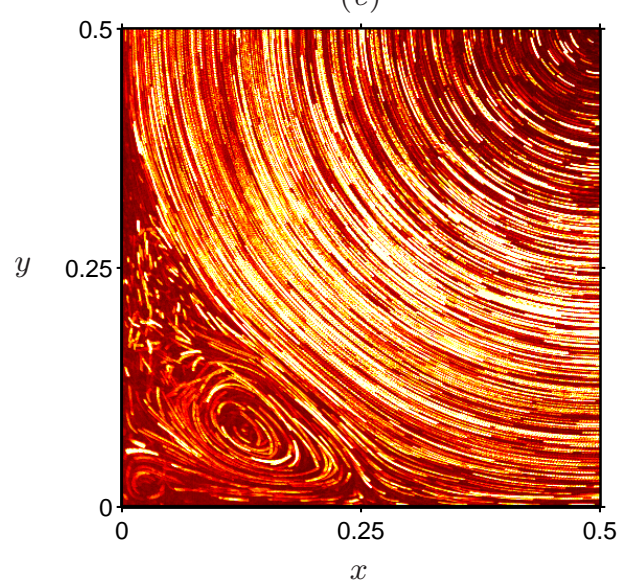

(b)

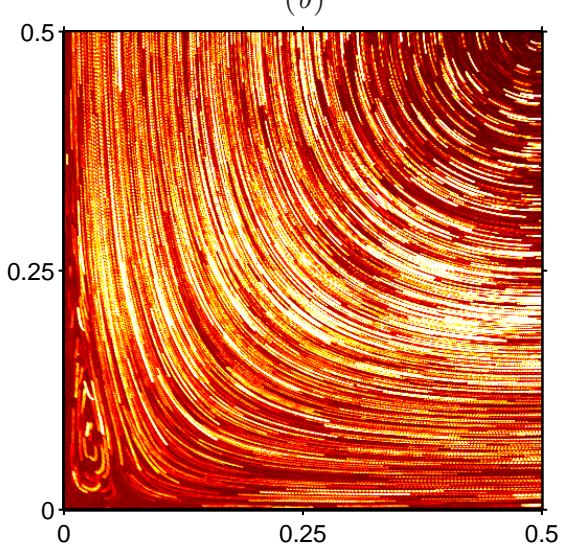

$(d)$

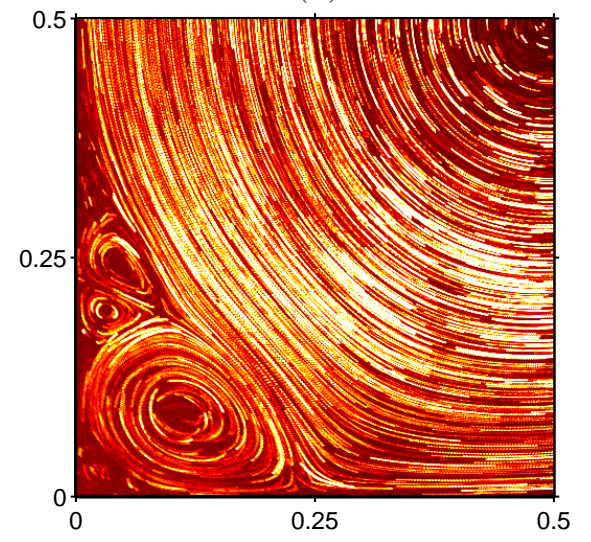

$(f)$

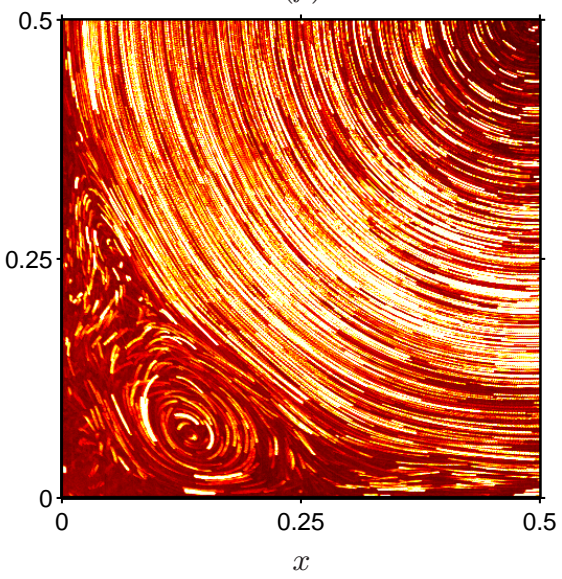

Figure 5. Experiment J: $R o=1, R e=13000, S=0, \Omega=0.091 \mathrm{rad} / \mathrm{s}, z_{0} / H=0.29$. Streakline images taken at dimensionless times $t=\Omega t^{*}=(a) 0.64,(b) 0.91,(c) 2.0,(d) 3.1,(e) 6.4,(f)$ 7.7 ; note that $t=2 \pi$ corresponds to the first rotation period of the turntable. The exposure time for each image was $3 \mathrm{sec}$. The black arrow in $(a)$ has been included to show the direction of the anticyclonic interior flow. 
primary vortex. At this stage, the four corner regions have deformed the interior starting flow, which now has a near-circular perimeter (a feature best illustrated by figure $2 d)$. As a result, there is now very little contact between the interior anticyclone and the container's sidewalls, and so the supply of cyclonic vorticity to the corners is now significantly reduced, and so there is little further growth of the corner regions.

The data in figures 2 and 4 are for the case where $S$ is large, with $R e$ at the lower end of the range considered. In this limit - corresponding to when the fluid's density stratification is 'strong' - the flow within the central core and the corner regions remains two-dimensional throughout the formation period. That is, in the absence of vertical motion, the illuminated tracer particles are advected along horizontal trajectories and so remain within the horizontal light sheet, resulting in continuous, well-defined streaklines. This is in contrast to the experiments performed with no density stratification, for $S=0$, which for the full range of $R e$ considered here were characterized by a period of threedimensional motion developing in the corner regions. This is best illustrated by comparing figure 4 with the streakline data in figure 5 , which is from an experiment performed under similar conditions $\left(R o=1, R e=13000, z_{0} / H=0.29\right)$, but with $S=0$. The key observation to highlight is that, during the initial period $0 \leqslant t \leqslant 3.1$ (i.e. the first half-rotation of the cylinder), the streakline patterns in figures $5(a)$ to $5(d)$ are identical to those in figures $4(a)$ to $4(d)$. That is, even at $S=0$, during this initial period the flow in both the central core and the corner region is two-dimensional. However, it is evident from figures $5(e)$ and $5(f)$ that shortly after this period the flow in the corner region becomes three-dimensional, with the tracer particles now moving vertically through, as well as in, the light sheet: The structure of the larger component of the divided primary vortex remains largely visible, but the structure of the weaker corner vortices is now unclear, because within these vortices the vertical and horizontal velocity components are comparable. (Note, the anticyclonic flow within the central core remains two-dimensional throughout.) For the experiment shown in figure 5, the three-dimensional motion was first evident at time $t \approx 4$. However, at time $t=10$, the vertical motion in the corner region had ceased, and flow throughout was again two-dimensional. We also note that in figures $5(e)$ and $5(f)$, the flow is three-dimensional, but not turbulent. It was only in experiments performed at the higher range values of $R e$ (above 37000) that the flow within the corner regions showed signs of transitioning to a fully turbulent state.

Most of the experiments reported here exhibited this period of three dimensional motion in the corner regions. It was only experiments at large $S$, or at intermediate values of $S$ with $R e$ at the lower end of the range considered, that the fluid's density stratification was strong enough to inhibit vertical motion, and in these cases the flow remained two-dimensional throughout the formation period. A key point worth highlighting, however, is that in all experiments, for all $S$ considered, the flow was always two-dimensional during the first half-rotation period (i.e. $0 \leqslant t \leqslant \pi$ ), and that the corner regions start forming during this initial period. In section 3 below, we introduce the theoretical analysis. In particular, the equations for the sidewall boundary layers are formulated, and the key assumption used is that, prior to break down, the sidewall boundary layers are two-dimensional. The experimental data presented above provides clear justification for this assumption.

It is also worth noting that the observations described above were confirmed by repeating a number of the experiments without the horizontal light sheet, but instead with the tracer particles illuminated uniformly. The particle motion was recorded using the digital camera set to view horizontally through the side of the cylinder, and focussed on one of the corner regions. In particular, these recordings confirmed the initial two- 
dimensionality of the flow, the columnar structure of the corner vortices, and the eventual onset of vertical motions in the corner regions (when $S$ was not large).

\section{A theoretical and numerical description}

The flow configuration has been discussed previously, and is shown in figure 1 . We present the theoretical analysis for the general case where the cylinder and fluid are initially in a state of solid rotation with angular frequency, $\Omega-\Delta \Omega$, and then at time $t^{*}=0$ the cylinder's rotation rate is increased instantaneously to $\Omega$. (The case of spin-up from rest corresponds to $\Omega=\Delta \Omega$.) To model the system, we make the usual Boussinesq approximation for the inclusion of density stratification, and write the equations of motion in a rotating frame of reference. Assuming the Froude number, $F r=\Omega^{2} L / g$, is small (in the experiments, $F r \leqslant 0.003)$, we neglect any modification of apparent gravity due to centrifugal acceleration and decompose the pressure and density into several components, so they can be written relative to the rotating coordinate system as

$$
\begin{gathered}
\bar{\rho}^{*}\left(\mathbf{x}^{*}, t^{*}\right)=\rho_{\ell}+\rho_{s}^{*}\left(z^{*}\right)+\rho^{*}\left(\mathbf{x}^{*}, t^{*}\right), \\
\bar{p}^{*}\left(\mathbf{x}^{*}, t^{*}\right)=p_{s}^{*}\left(z^{*}\right)+p^{*}\left(\mathbf{x}^{*}, t^{*}\right),
\end{gathered}
$$

where $\rho_{s}^{*}$ and $p_{s}^{*}$ are the contributions associated with the background stratification, $\rho_{\ell}$ is a constant reference density (taken here to be the initial density at the container lid), and $\rho^{*}$ and $p^{*}$ are the perturbations arising due to the increase in rotation rate of the cylinder. It is assumed throughout that $\rho^{*} \ll \rho_{s}^{*}, \rho_{\ell}$.

Following Foster \& Munro (2012), we introduce the non-dimensional variables

$$
\mathbf{x}=\frac{\mathbf{x}^{*}}{L}, t=\Omega t^{*}, \mathbf{u}=\frac{\mathbf{u}^{*}}{\Delta \Omega L}, \rho=\frac{g \rho^{*}}{\rho_{\ell} \Omega \Delta \Omega L}, p=\frac{p^{*}}{\rho_{\ell} \Omega \Delta \Omega L^{2}} .
$$

The governing equations of motion are therefore reduced to

$$
\begin{gathered}
\nabla \cdot \mathbf{u}=0 \\
\mathbf{u}_{t}+R o(\mathbf{u} \cdot \nabla) \mathbf{u}+2(\hat{\mathbf{z}} \times \mathbf{u})+\rho \hat{\mathbf{z}}+\nabla p=E \nabla^{2} \mathbf{u} \\
\rho_{t}+\operatorname{Ro}(\mathbf{u} \cdot \nabla) \rho-S w=(E / \sigma) \nabla^{2} \rho,
\end{gathered}
$$

where the dimensionless Rossby $(R o)$, Ekman $(E)$, Burger $(S)$ and Schmidt $(\sigma)$ numbers are, as noted above, defined by

$$
R o=\frac{\Delta \Omega}{\Omega}, E=\frac{\nu}{\Omega L^{2}}=\frac{R o}{R e}, S=\left(\frac{N}{\Omega}\right)^{2}, \sigma=\frac{\nu}{\kappa} .
$$

We assume that the buoyancy frequency $N$ remains constant throughout. The case where the fluid has uniform density is governed by (3.3) with $\rho=0, S=0$. The case of spin-up from rest corresponds to $R_{o}=1$.

The initial and boundary conditions for (3.3) are

$$
\begin{gathered}
\mathbf{u}=\hat{\mathbf{z}} \times\left(\mathbf{x}-\mathbf{x}_{0}\right)=\left(\frac{1}{2}-y, x-\frac{1}{2}, 0\right), \rho=0 \text { for } t=0, \mathbf{x} \in \mathcal{D}, \\
\mathbf{u}=0, \frac{\partial \rho}{\partial n}=0 \text { for } \mathbf{x} \in \partial \mathcal{D}, t>0,
\end{gathered}
$$

where $\mathbf{x}_{0}$ denotes the position vector of the cylinder-base centre, $\mathcal{D}=\{(x, y, z): x, y \in$ $[0,1], z \in[0, h]\}$ is the flow domain, $h=H / L$ is the cylinder's height-to-width ratio, $\partial \mathcal{D}$ the domain boundary, and $n$ is used to represent the normal direction at each boundary. 


\subsection{The two-dimensional response over the $\mathcal{O}\left(R o^{-1} \Omega^{-1}\right)$ timescale}

The recent work of Foster \& Munro (2012) showed that there is no eddy generation in the sidewall region (adjacent to $\partial \mathcal{D}_{v}$, which denotes the vertical parts of $\partial \mathcal{D}$ ) for sufficiently small changes in rotation rate. The asymptotic restriction for this scenario is $R o \ll E^{1 / 2}$, and in such cases, the sidewall region remains a growing Rayleigh layer on the spin-up timescale. Furthermore, when $R o=O\left(E^{1 / 2}\right)$, corner eddies were observed on the spin-up timescale $E^{-1 / 2} \Omega^{-1}$, and it was speculated that their origin lay in a break down/eruption of a viscous boundary layer, owing to an adverse pressure gradient imposed via the interior starting flow. At these (and indeed larger) Rossby numbers, Foster \& Munro (2012) showed that the sidewall region requires a solution of the (nonlinear) Prandtl boundary-layer equations, which certainly allows for the possibility of a break down. In the linear regime, the influence of the top and bottom Ekman layers remains important to the core flow structure, but fortunately, in the nonlinear regime, Ekman suction is not important to the leading-order dynamics. As a consequence, for $R o \gg E^{1 / 2}$ the timescale for evolution of the boundary layer is short compared to the interior spin up timescale and the motion in the core may be treated as quasi-steady and two-dimensional, at least up to the point of an eruption from sidewall viscous layers. In Appendix A we have provided a theoretical justification for treating the flow as two-dimensional during this initial period.

The sidewall boundary layers are crucial in the nonlinear regime, and of more significance than the top and bottom Ekman layers in stratified flow. Both Foster \& Munro (2012) and van Heijst et al. (1990) have speculated that a break down of such boundary layers is the origin of columnar eddies, but the evolution of the viscous sidewall region has not been explicitly considered. Given the parabolic nature of the boundary layer on $\partial \mathcal{D}_{v}$, the problem is made non-trivial by the periodic nature of the layers across the four boundaries of the container cross section. Furthermore, we expect the sidewall region to become bi-directional (that is, develop recirculatory regions). Clearly the nature of the flow in the connecting corners $(x, y)=(0,0),(1,0),(1,1)$ and $(0,1)$ will be of some significance.

We begin by considering, for example, the boundary layer on the $y=0^{+}$wall with $x \in[0,1]$. We first introduce a scaled time $t=R o^{-1} \bar{t}$, a transverse boundary-layer coordinate $y=(E / R o)^{\frac{1}{2}} \bar{y}$ and let $v=(E / R o)^{\frac{1}{2}} \bar{v}$, so that the $x$-direction momentum equation becomes

$$
u_{\bar{t}}+u u_{x}+\bar{v} u_{\bar{y}}+Q_{x}(x, z, \bar{t})=u_{\bar{y} \bar{y}}
$$

as discussed in section 4 of Foster \& Munro (2012) and Appendix A. Note that for spinup from rest, which is the focus of the experimental results presented earlier, $R_{0}=1$ so that $t=\bar{t}$. Here $Q(x, z, \bar{t})$ is a pressure correction such that $Q_{x}=-U U_{x}$, where $U$ is the external flow speed along $y=0^{+}$, which is (initially at least) determined from the inviscid starting flow (2.3). In the most general case, the outer flow along $y=0^{+}$ could be dependent on $z$ (and indeed $\bar{t}$ ) as well as $x$, however prior to eruption of the side wall layers the $z, \bar{t}$ dependence is trivial, apart from locations very near the top and bottom boundaries, so we take $U=U(x)$. However, if there is a singular break down of (3.6) at finite $\bar{t}=\bar{t}_{s}$, then the assumption of a simple quasi-steady interior flow must be abandoned for $\bar{t}>\bar{t}_{s}$.

Prior to any eruption, with $U=U(x)$ determined by (2.3) with $y=0$, we can solve for the evolution of the sidewall boundary layer on $y=0^{+}$. In terms of a streamfunction formulation, with $u=\bar{\psi}_{\bar{y}}$ and $\bar{v}=-\bar{\psi}_{x}$, the boundary layer system (3.6) reduces to

$$
\bar{\psi}_{\bar{y} \bar{t}}+\bar{\psi}_{\bar{y}} \bar{\psi}_{\bar{y} x}-\bar{\psi}_{x} \bar{\psi}_{\bar{y} \bar{y}}=U(x) U^{\prime}(x)+\bar{\psi}_{\bar{y} \bar{y} \bar{y}},
$$


subject to the boundary conditions $\bar{\psi}=\bar{\psi}_{\bar{y}}=0$ on $\bar{y}=0$ and $\bar{\psi}_{\bar{y}} \rightarrow U(x)$ as $\bar{y} \rightarrow \infty$.

It is important to note that, along $y=0$, near the corner $(x=0)$ the exterior velocity is such that

$$
U(x) \sim-\frac{4}{\pi} x \log x
$$

and similarly near $x=1$,

$$
U(x) \sim-\frac{4}{\pi}(1-x) \log (1-x)
$$

This follows from an analysis of $(2.2)$ in terms of a polar coordinate system $(r, \theta)$ centred at a corner, as $r \rightarrow 0$. The response for small $r$ is dominated by the constant vorticity term, which leads to the logarithmic response. This free stream behaviour leads to a rather more complicated boundary layer formulation, and is best dealt with via a rescaling:

$$
Y=\left(\frac{U(x)}{x(1-x)}\right)^{\frac{1}{2}} \bar{y}, \quad \tau=\frac{U(x)}{x(1-x)} \bar{t}, \quad \bar{\psi}=(x(1-x) U(x))^{\frac{1}{2}} \Psi(x, Y, \tau) .
$$

For the boundary layer computation we consider an idealised impulsive transition of the container rotation rate. To capture this short-time impulsive behaviour we make use of a further rescaling by introducing a Rayleigh-layer coordinate $Y=\tau^{\frac{1}{2}} \hat{Y}$, where $\Psi(x, Y, \tau)=\tau^{\frac{1}{2}} \hat{\Psi}(x, \hat{Y}, \tau)$. The side-wall equation in this rescaled formulation then becomes

$$
\begin{aligned}
& \hat{\Psi}_{\hat{Y} \hat{Y} \hat{Y}}+\frac{\hat{Y}}{2} \hat{\Psi}_{\hat{Y} \hat{Y}}-\tau \hat{\Psi}_{\hat{Y} \tau}+ \\
& \tau x(1-x)\left\{\hat{\Psi}_{\hat{Y} \hat{Y}} \hat{\Psi}_{x}-\hat{\Psi}_{\hat{Y}} \hat{\Psi}_{\hat{Y} x}\right\}+\tau^{2} \lambda(x) x(1-x)\left\{\hat{\Psi}_{\hat{Y} \hat{Y}} \hat{\Psi}_{\tau}-\hat{\Psi}_{\hat{Y}} \hat{\Psi}_{\hat{Y} \tau}\right\} \\
& \quad=\frac{\tau x(1-x) U^{\prime}(x)}{U(x)}\left\{\hat{\Psi}_{\hat{Y}}^{2}-1\right\}+\tau(2 x-1) \hat{\Psi} \hat{\Psi}_{\hat{Y} \hat{Y}},
\end{aligned}
$$

where

$$
\lambda(x)=\frac{U^{\prime}(x)}{U(x)}+\frac{2 x-1}{x(1-x)},
$$

which remains regular in the limit of $\tau \rightarrow 0$.

\subsection{The limiting boundary layer solution, on approaching a corner}

If we consider (3.10) as $x \rightarrow 0^{+}$and $x \rightarrow 1^{-}$, when neglecting terms of $\mathcal{O}(1 / \log (x))$ and $\mathcal{O}(1 / \log (1-x))$ respectively, we find that

$$
\hat{\Psi}_{\hat{Y} \hat{Y} \hat{Y}}+\frac{\hat{Y}}{2} \hat{\Psi}_{\hat{Y} \hat{Y}}-\tau \hat{\Psi}_{\hat{Y} \tau}= \pm \tau\left\{\hat{\Psi}_{\hat{Y}}^{2}-1-\hat{\Psi} \hat{\Psi}_{\hat{Y} \hat{Y}}\right\}
$$

is the leading-order balance, where the positive sign is taken at $x=0$ and the negative at $x=1$.

The governing equation is clearly different at $x=0^{+}$and $x=1^{-}$, which suggests that there is a marked difference between the boundary layer profiles on the "incoming' and 'outgoing' sides of each corner. Furthermore, the solution near $x=0^{+}$approaches a steady state, whilst that near $x=1^{-}$thickens with increasing $\tau$. Nevertheless, at finite values of the computational 'time' $\tau$, the boundary layer remains thin, of $\mathcal{O}\left(R e^{-1 / 2} / \log (x)\right)$ near $x=0^{+}$and $\mathcal{O}\left(R e^{-1 / 2} / \log (1-x)\right)$ near $x=1^{-}$, where $R e=R o / E$ is a Reynolds number. This suggests that a self-consistent leading-order 

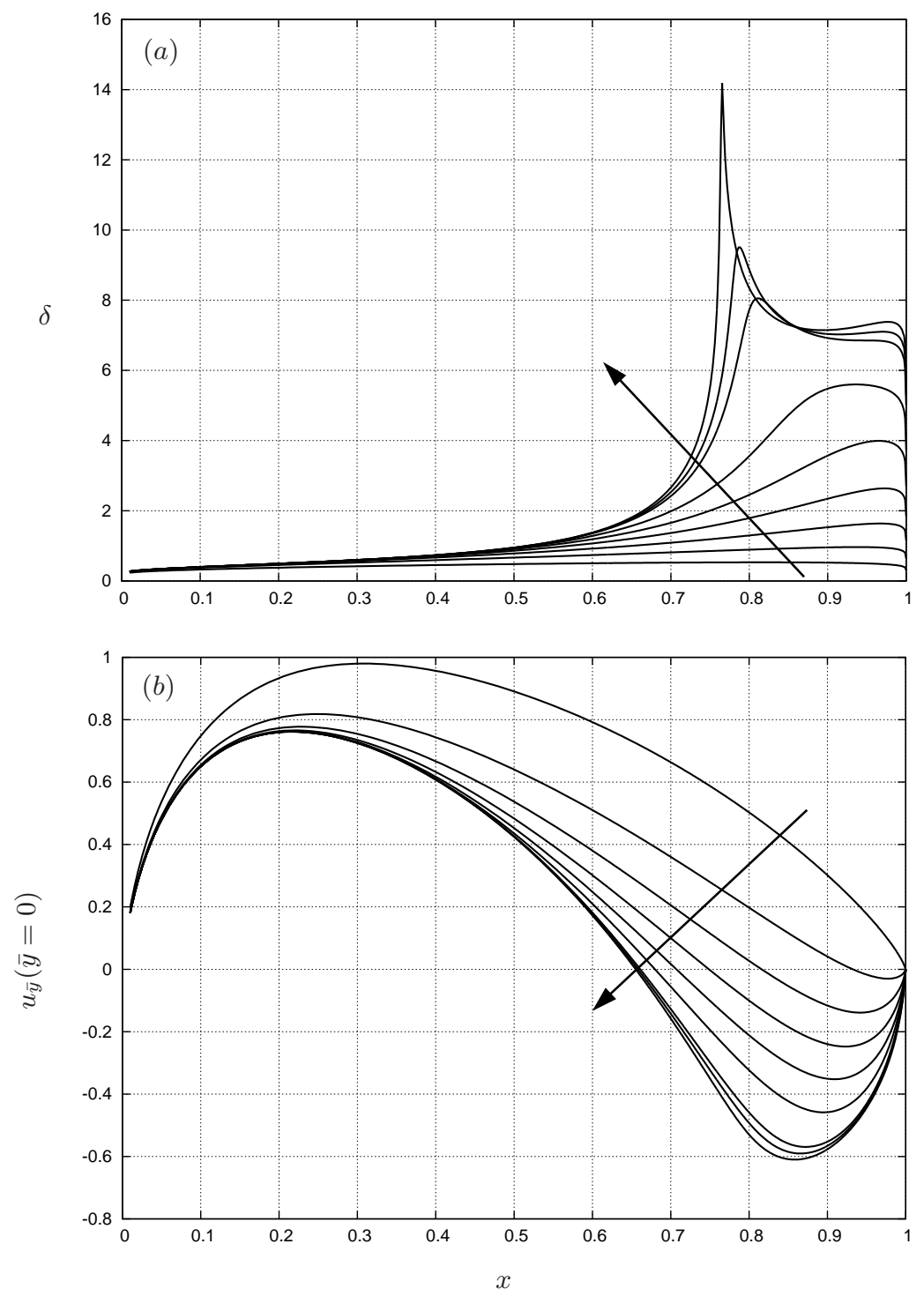

FIGURE 6. Boundary layer results: (a) The evolution of the displacement thickness, $\delta$, at re-scaled computational times of $\tau=0.5,1, \ldots, 3.5,3.6,3.7$, (b) The evolution of the shear distribution along the lower wall $\bar{y}=0$, at re-scaled computational times of $\tau=0.5,1, \ldots, 3.5,3.6,3.7$. In both figures $\tau$ increases in the direction of the arrow shown.

asymptotic approach can be constructed for high Reynolds number, for which the inflow and outflow conditions at each of the four corners remain decoupled when considering the boundary layer evolution. We will check this formulation later, by quantitative comparisons to a comparable two-dimensional Navier-Stokes formulation for finite values of Re.

\subsection{Unsteady boundary-layer solutions}

The side wall evolution can now be determined by a solution of (3.10) subject to $\hat{\Psi}=$ $\hat{\Psi}_{\hat{Y}}=0$ on $\hat{Y}=0$ and $\hat{\Psi}_{\hat{Y}} \rightarrow 1$ as $\hat{Y} \rightarrow \infty$. Initial conditions for the boundary-layer 


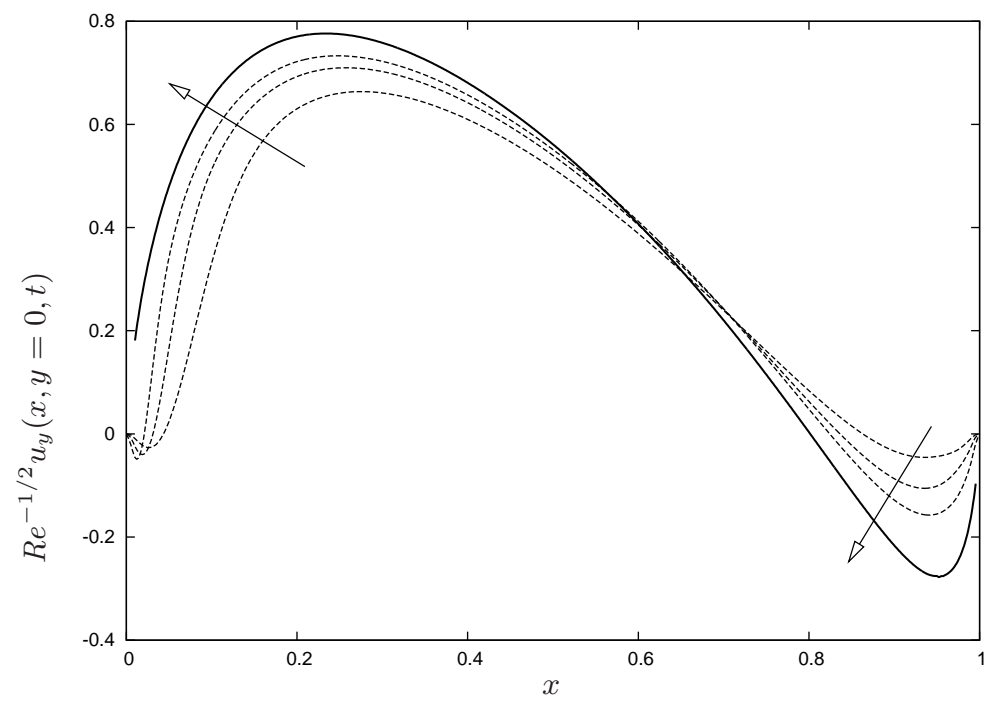

Figure 7. A comparison of boundary layer results with two-dimensional Navier-Stokes computations. Profiles of scaled wall shear $R e^{-1 / 2} u_{y}(x, y=0, \bar{t})$ are shown at $\bar{t}=0.5$. Navier-Stokes results for $R e=1250,5000$ and 20000 are shown as dashed lines (Re increasing in the direction of the arrows). The prediction of the boundary-layer computation is also shown as the solid line. For spin-up from rest $R o=1$ and so $\bar{t}=t=\Omega t^{*}$.

system are simply the Rayleigh layer solution, as obtained from (3.10) with $\tau=0$. Along $y=0$ the anticyclonic interior flow is in the direction of increasing $x$, therefore to complete the specification of the parabolic side-wall boundary-layer system, we need the starting velocity profile that arises in the corner $x=0^{+}$. To obtain this starting profile we solve the unsteady one-dimensional boundary-value problem obtained from (3.11). Although the interior motion is always from $x=0^{+}$to $x=1^{-}$, the flow within the boundary layer may reverse near to the wall owing to the adverse pressure gradient associated with the interior starting flow. When the boundary layer contains such a recirculation region, we must also specify the comparable solution as $x \rightarrow 1^{-}$, which again can be determined from (3.11).

Our computational procedure solves (3.10) via a second-order box scheme. To cope with the logarithmic corrections near the corners we employ a comparable rescaled (logarithmic) downstream coordinate. To cope with flow reversal we make use of the 'zig-zag' modification of the box scheme (Cebeci 1986), which necessitates the inclusion of the solution at $x=1^{-}$as noted above.

In terms of the unscaled streamwise velocity $u(x, y, t)$, for the boundary layer adjacent to $y=0$, the displacement thickness is

$$
\delta(x, t)=\int_{\bar{y}=0}^{\infty}(1-u / U(x)) \mathrm{d} \bar{y},
$$

where $U(x)$ is the 'free stream' velocity associated with the inviscid core flow along the wall. In terms of the rescaled transverse coordinate $(\hat{Y})$, stream function $(\hat{\Psi})$ and time $(\tau)$, this becomes

$$
\delta(x, \tau)=\left(\frac{x(1-x) \tau}{U(x)}\right)^{\frac{1}{2}} \int_{\hat{Y}=0}^{\infty}\left(1-\hat{\Psi}_{\hat{Y}}(x, \tau)\right) \mathrm{d} \hat{Y} .
$$

In figure 6 we show the evolution of this displacement thickness, $\delta(x, \tau)$, and wall shear, 

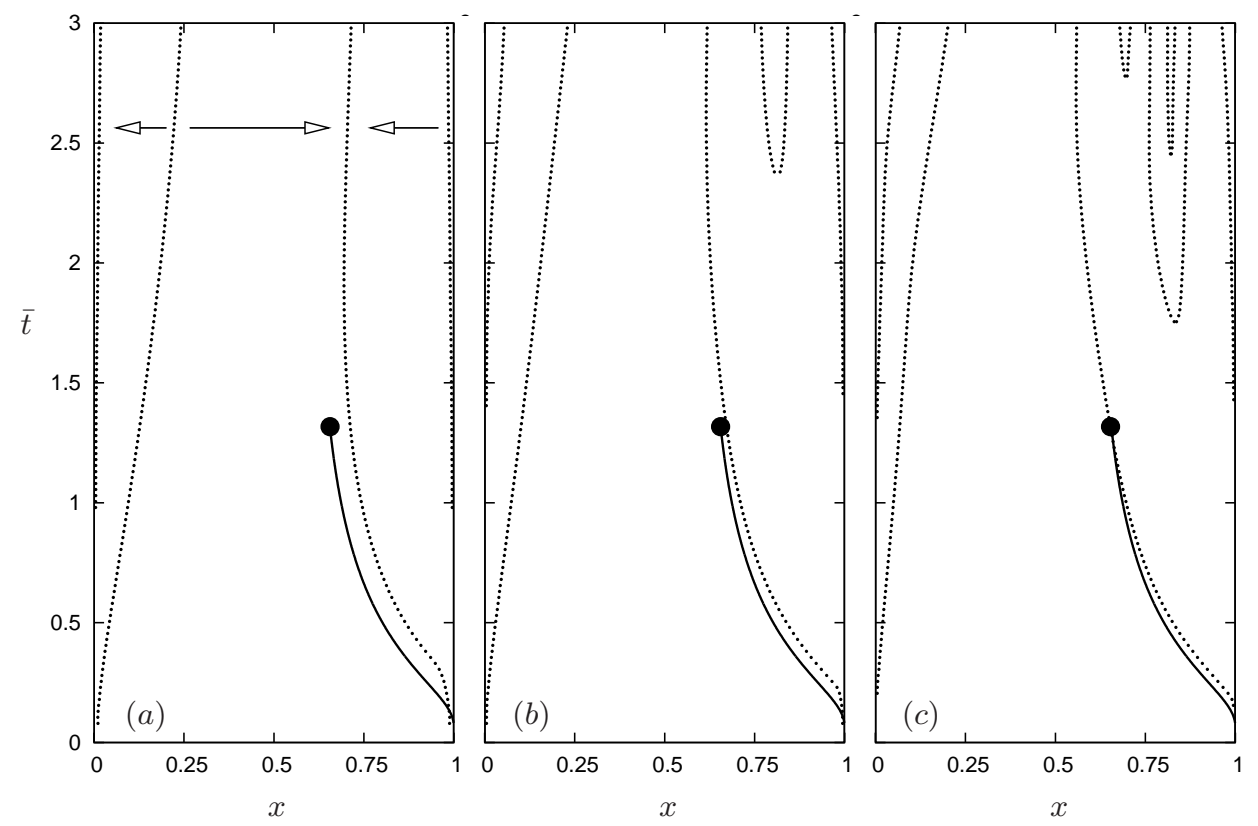

Figure 8. The loci of points of zero shear, $u_{y}(x, y=0, \bar{t})=0$, along the $y=0$ wall at finite Reynolds numbers for a two-dimensional Navier-Stokes computation, which shows the development of the columnar corner eddies at (a) $R e=1250$, (b) $R e=5000$ and (c) $R e=20000$. The solid line indicates the extent of the corner eddy, as predicted by boundary-layer theory, which terminates with a singular break down near $\bar{t} \approx 1.25$, indicated by the filled circle. The arrows in (a) indicate the sense of the flow direction adjacent to $y=0$, left-to-right being anticyclonic and right-to-left being cyclonic. For spin-up from rest $R o=1$ and so $\bar{t}=t=\Omega t^{*}$.

$u_{\bar{y}}(x, \bar{y}=0)$, at constant values of the re-scaled computational time $\tau$. Reverse flow is seen to develop first at the right-hand corner $x=1$ around $\tau \approx 1$. This reverse flow region grows, with the location of the zero wall shear moving 'upstream' (i.e. to the left) towards $x \approx 0.65$. The flow along the left-hand half $(x<0.5)$ of the lower $(y=0)$ boundary is essentially quasi-steady by the time the point of vanishing shear has advanced to $x<0.7$. However, in the recirculation region a spike in displacement thickness is found to occur at a finite-time, near $x \approx 0.75$. This singular displacement thickness is associated with an eruption of the side wall boundary layer into the bulk fluid at $R o \Omega t^{*} \approx 1.25$, and for larger times the boundary-layer approximation is obviously no longer valid.

\subsection{A two-dimensional finite Re formulation}

We now consider a direct computation of the Navier-Stokes equations at large, but finite, Reynolds number in order to examine how the predictions arising from the boundarylayer theory are realised in the full equations. We will still assume that the flow remains two-dimensional on the timescale of interest, and compare the predicted flow structure to the experimental results obtained for spin-up from rest $(R o=1)$. The Navier-Stokes equations are solved using an adaptive, Galerkin finite element method implemented via the library oomph-lib, see Heil \& Hazel (2006). The computational domain is now the entire cross-section of the container, and we do not assume any symmetry in the system. The fluid variables are discretised using isoparametric, $Q_{2} P_{1}$ (Taylor-Hood) elements in which the velocities are interpolated quadratically and the pressures are interpolated linearly within each element. The time derivative terms are treated implicitly using a 

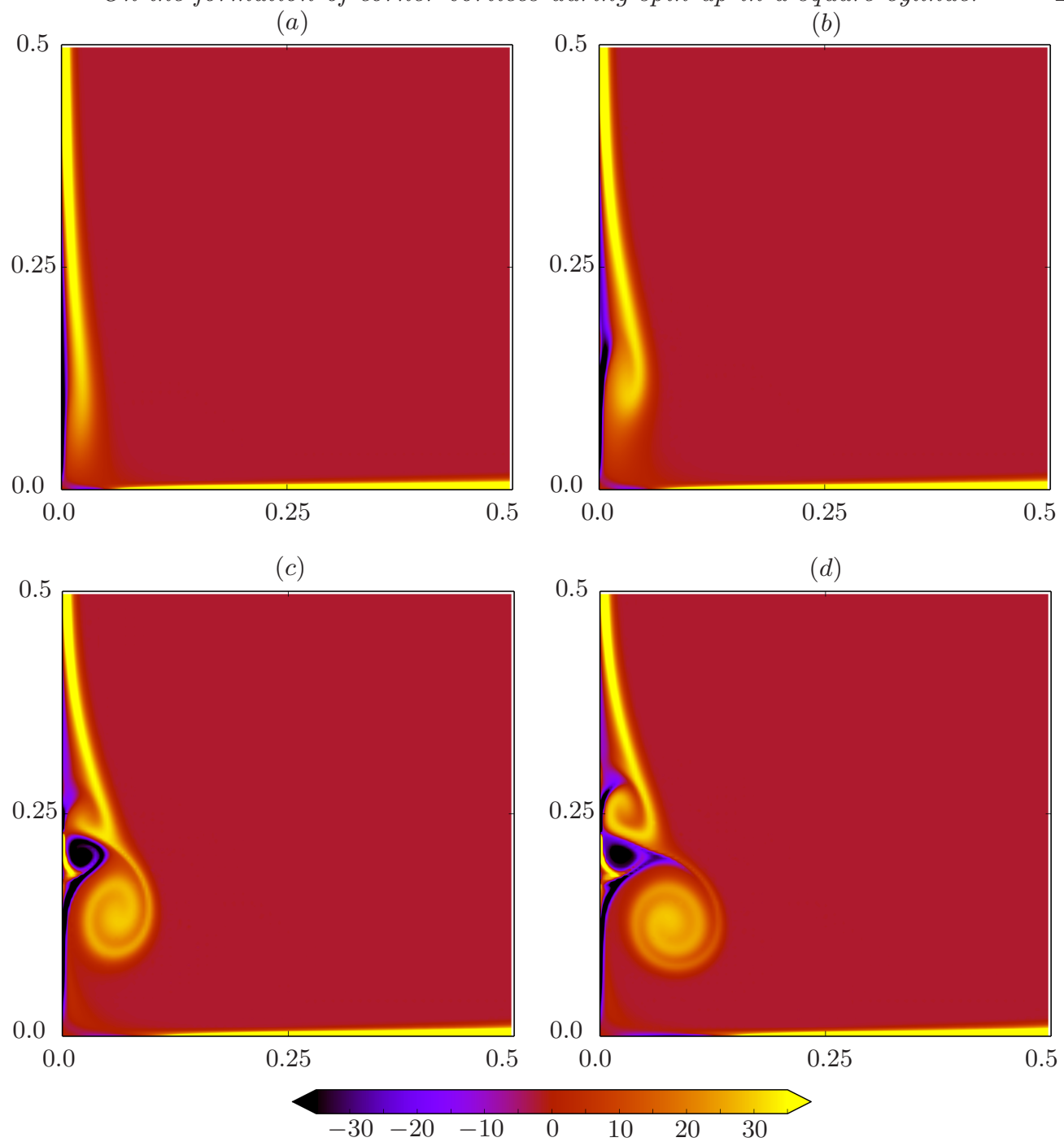

FIGURE 9. Vorticity contours obtained from a two-dimensional Navier-Stokes computation, for spin-up from rest with $R e=40000$ at $\bar{t}=1.25,1.65,2.25$ and 2.5 for (a) to (d) respectively. Contours are uniformly spaced between values of -35 to 35 . For spin-up from rest $R o=1$ and so $\bar{t}=t=\Omega t^{*}$.

second-order BDF2 method and the resulting discrete nonlinear system is solved by Newton iteration.

Our boundary-layer analysis is developed assuming an impulsive transition in the rotation rate of the container. For the Navier-Stokes computation we use initial conditions obtained from a composite solution constructed from the inviscid solution (2.3), modified to match the required no-slip conditions at the boundary using the small- $\tau$ boundary layer solution. For the BDF2 method, this composite solution is assumed at times of $t=\Delta t$ and $t=2 \Delta t$ (where $\Delta t$ is the computational time step, typically 0.005 ) then computation determines the subsequent evolution for $t=n \Delta t, n=3,4, \cdots$. This approach has been used in other comparable impulsive boundary-layer problems with some success, such as Hewitt et al. (2011). Spatial adaptivity is employed to accurately resolve the 
fine near-wall structures and results presented herein have been confirmed to be converged by repeated computation over different spatial-temporal refinements. Typically the computation had approximately $2.5 \times 10^{5}$ degrees of freedom.

\subsubsection{A comparison to boundary layer theory}

In figure 7 we begin by comparing the wall shear along $y=0$ at a fixed time of $\bar{t}=0.5$ and increasing Reynolds number $R e=R o / E=1250,2500$ and 20000. At this time, $\bar{t}=R o \Omega t^{*}=0.5$, the boundary layer has a single recirculation region, as suggested by the negative wall shear region near $x=1$, and there is good agreement between the finiteRe numerical results and the asymptotic (boundary layer) theory for increasing $R e$. In figure 8 we track the location of the zero shear points along the wall $y=0$ as a function of dimensionless time $\bar{t}=R o \Omega t^{*}$. Figures 8 (a-c) show that multiple recirculations develop. At finite Reynolds number, the flow is initially anticyclonic near $y=0$, with a thin developing region of cyclonic flow found near the corner corresponding to $x=0$ and $x=1$. This cyclonic corner eddy grows in extent, a growth that continues until two further sub-regions of anticyclonic motion develop, one appears further into the corner, whilst the other can be seen near $x \approx 0.8$ (see figure $8 \mathrm{c}$ ).

As the Reynolds number is increased, the growth of the primary (cyclonic) corner eddy is in line with the boundary-layer prediction as shown by the solid line in figure 8 . The time of the boundary layer eruption is shown in figure 8 by the circular datum point near $\bar{t}=\bar{t}_{s} \approx 1.25$. Although the boundary-layer solution terminates with the eruption, at finite Reynolds numbers this singular behaviour is rapidly mitigated as the boundary layer thickens.

Finally, in figure 9 we show the evolution of the vorticity field for $R e=40000$. For asymptotically high Reynolds numbers the (attached, non-interacting) boundary layer only exists up to $\bar{t} \approx 1.25$, but the separation is delayed by finite values of $R e$. In figure 9 (a) the boundary layer is showing signs of thickening near $x=0, y=0.1$ at $\bar{t}=1.25$. The location of this thickening is propagating to larger values of $y$, against the dominant motion of the anticyclonic core flow, and by $\bar{t}=1.65$ has become much more prominent at $y \approx 0.15$. Shortly afterwards, at $\bar{t}=2.25$, there is clearly no simple attached wall layer, having separated near $y \approx 0.25$. At this latest time value, the container has undergone approximately one quarter of its first revolution in the context of spin-up from rest.

\subsubsection{A comparison to laboratory data}

Having established good quantitative agreement between the two-dimensional NavierStokes calculations and the unsteady boundary-layer theory, we now move on to comparing against data acquired in the experimental work. As the numerical solution assumes an impulsive transition of the container rotation frequency, the computational times for the numerical results are measured from when the container frequency (of the laboratory experiment) is at its final value of $0.083 \mathrm{rad} / \mathrm{s}$.

We first present a qualitative comparison, by showing instantaneous streamlines of the numerical solution at the same times for which experimental results are shown in figure 4(a-f) (which are for the case $R o=1, R e=12000$ ). Figure 10 shows the corresponding two-dimensional finite Reynolds number predictions for $R e=12000$; here (a-f) are to be compared to the respective frames of figure 4 .

There is a good correspondence between the numerical results of figure 10 and the experimental visualisation of figure 4. However, for a more quantitative comparison we also examine the predicted and measured horizontal velocity components $(u, v)$ along arbitrary cross sections of the domain. Figure 11 shows both $u(x, y, t)$ and $v(x, y, t)$ as functions of $x$ at fixed times $t=1.1,3.2,7.8$ (corresponding to those shown in figures 10 

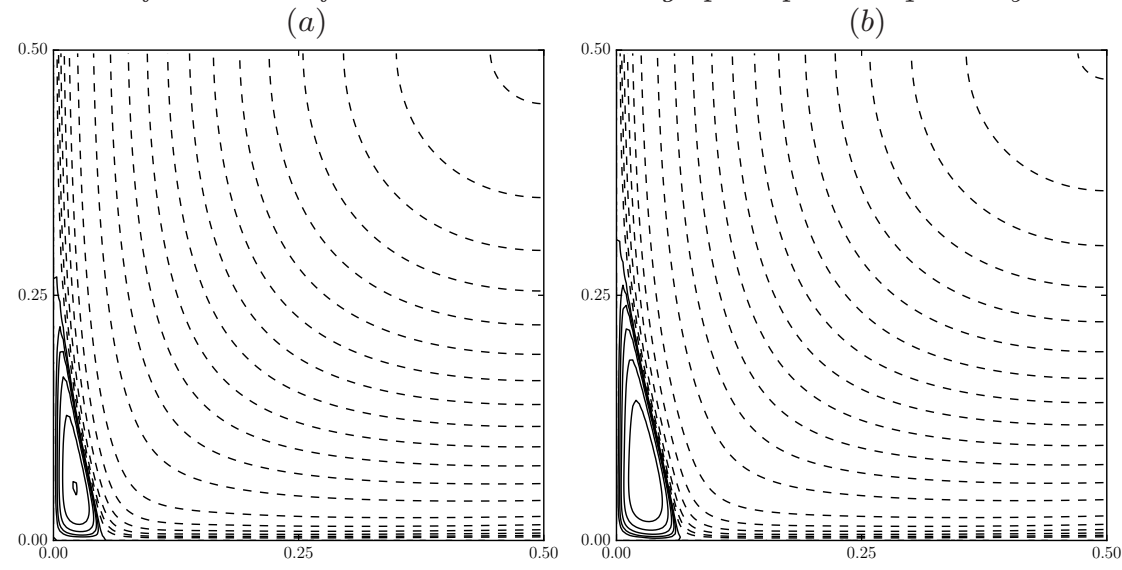

(c)
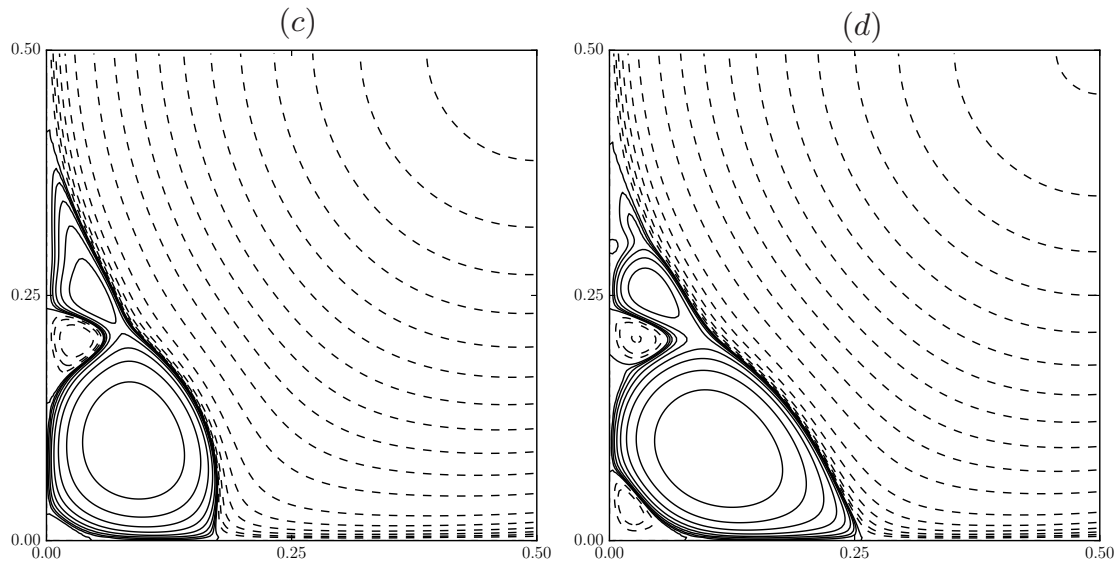

$(e)$
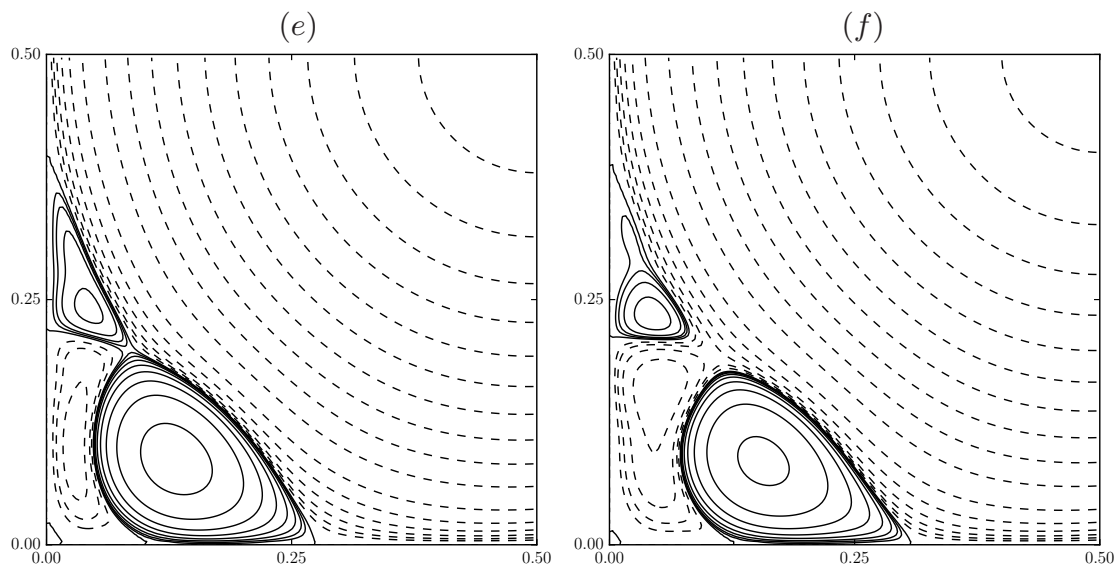

Figure 10. Results of a Navier-Stokes computation with $R e=12000$, assuming a two-dimensional response. The contours of the streamfunction are shown at times that correspond to the $(R o=1)$ experimental images of figure 4(a)-(f) respectively. Contours are non-uniformly distributed at values of $0,-0.001,-0.002,-0.004, \ldots,-0.018$ (solid line, cyclonic) and $0.15,0.14,0.13, \ldots, 0.01,0.005,0.0025, \ldots, 0.0003125$ (dashed lines, anticyclonic). 

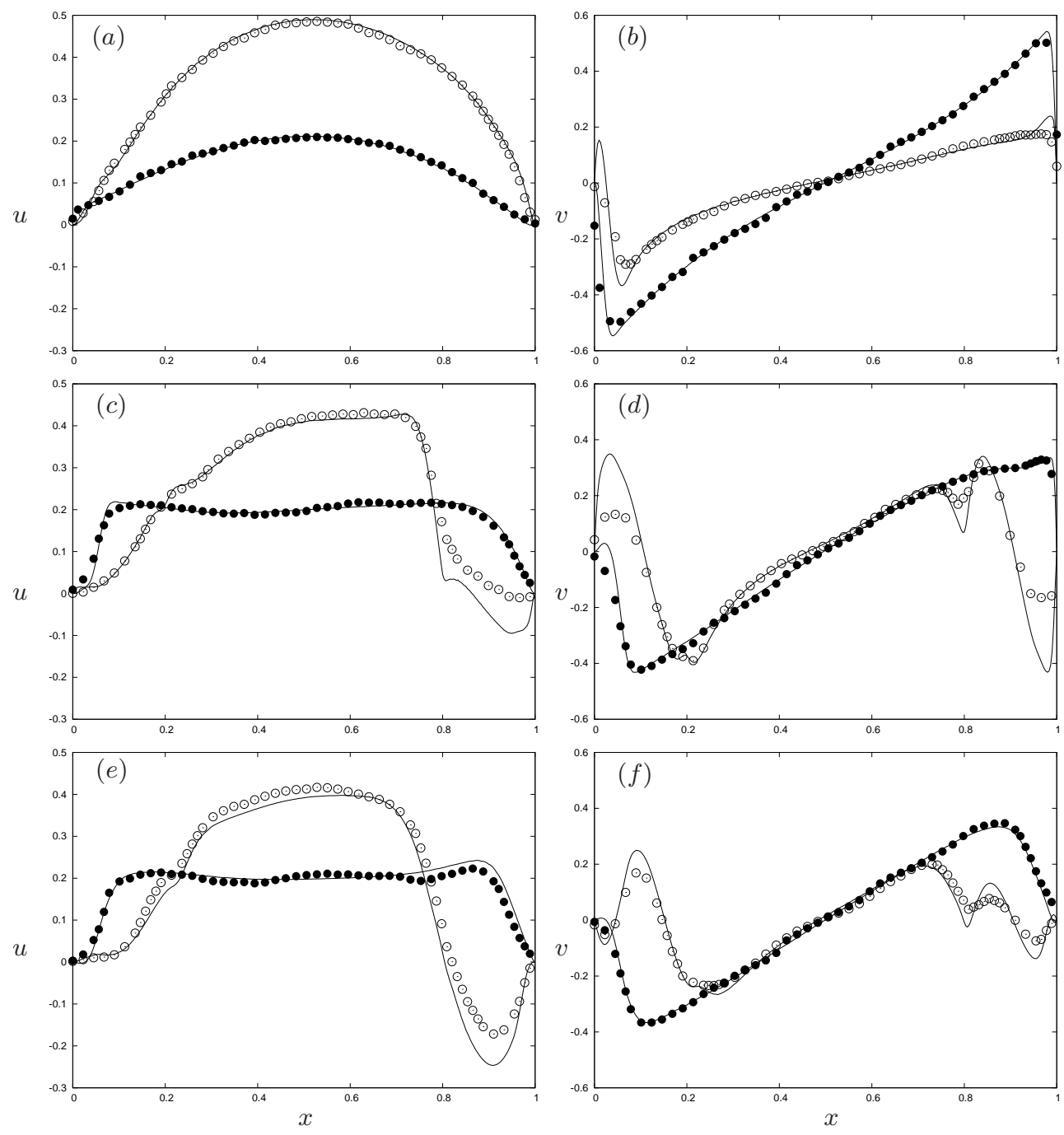

FiguRE 11. A comparison of experimental results with a two-dimensional Navier-Stokes computation. Horizontal velocity components are shown at constant values of $y$, for the experiment shown in figure 4 . The component $u(x, y, t)$ is shown in (a) $t=1.1$, (c) $t=3.2$ (e) $t=7.8$ and $v(x, y, t)$ is shown in (b) $t=1.1$, (d) $t=3.2$ (f) $t=7.8$. The open data points correspond to experimental data acquired at $y=0.1$ whilst the filled data points are acquired at $y=0.3$. The solid lines are the corresponding results obtained from the two-dimensional Navier-Stokes solution shown in figure 10.

and 4$)$ and $y=0.1,0.3$. These $y$ locations are chosen to be both in the bulk of the corner vortex, and at its outer edge when fully developed. There is a good quantitative correspondence between the experimentally acquired velocity profiles and the two-dimensional prediction. 


\section{Discussion}

We have presented the results of an experimental and theoretical investigation into the spin-up of a fluid (homogeneous or linearly stratified) in a cylinder of square cross section. Over the time it takes to perform one rotation of the container, the Ekman layers on the top and bottom boundaries are still developing, but the sidewall layers separate from the (vertical) container walls. This separation leads to an eruption of vorticity and axial vortices becoming trapped in the vertical corners of the container, leaving an approximately circular separating streamline for the anti-cyclonic interior flow. The work of Foster \& Munro (2012) showed that these sidewall layers are governed by the (nonlinear) Prandtl boundary layer equation and in this work we explicitly show that a solution of the boundary-layer system does indeed reveal a singular breakdown (at $R o \Omega t^{*} \approx 1.25$ ) as conjectured previously by van Heijst et al. (1990). Beyond this critical time an attached (non-interacting) boundary-layer solution is not possible, as the displacement thickness becomes unbounded.

The evolution of the boundary layer has qualitative similarities to the impulsive translation of a cylinder in a viscous incompressible fluid; see for example Koumoutsakos \& Leonard (1995). In the context of the flow adjacent to the boundary $y=0, x \in(0,1)$, as described in section 3 , we may conceptually associate the $x=0^{+}, 1^{-}$positions with the forward/rear attachment points respectively in the cylinder problem. Near $x=0^{+}$ a quasi-steady solution is established, but near $x=1^{-}$the boundary layer continues to thicken. The thickening of the boundary layer propagates upstream, until a singular eruption is achieved in a finite time. What makes this problem somewhat unusual is that periodicity of the square container means that $x=1^{-}$solution is connected to the $x=0^{+}$solution via the corner regions of the container; the external flow also contains a logarithmic dependence in the corner regions. Nevertheless, at finite values of the rescaled time $(\tau)$ appropriate to the boundary layer evolution, and in the leading order high- $R e$ limit, the layers are formally empty at the corners, albeit only logarithmically so. This allows for a consistent high-Reynolds number asymptotic description.

Given the unusual periodic nature of the boundary layer, we have been careful to validate the asymptotic description with a finite Reynolds number numerical solution, both of which assume a two-dimensional response over a timescale of $\mathcal{O}\left(R o^{-1} \Omega^{-1}\right)$. The analysis of Appendix A, together with the observations and data from the experimentsperformed for the case of spin-up from rest, $R o=1$-justify this assumption, showing that the flow (in the corners and the central core) remains horizontally two-dimensional during the period prior to, and shortly after, the singular break down of the side wall layers. Very good agreement is found between the high-Re boundary layer and the twodimensional Navier-Stokes predictions. In addition, the qualitative nature of the instantaneous streamlines and the quantitative comparison of laboratory data both show remarkable agreement with these same two-dimensional numerical predictions for the case $R o=1$. When $S$ is large - corresponding to a strongly stratified fluid - this agreement persists over one rotation of the container, as shown in figure $4(S=100)$ and figure 10, but as $S$ is reduced, three-dimensional flow becomes apparent in the corner vortices after approximately half a rotation of the container.

\section{Appendix A. More on two-dimensionality}

The equations of motion are given as (3.3). As noted in $\S 3.1$, a key issue is the twodimensionality of the flow. At least prior to the eruption of side-wall vorticity into the core, it is convenient to consider the inviscid core and the side-wall boundary layers 
separately on this point. It will emerge that such considerations lead to more insight into the subsequent motion as well. Below, we consider the interior motion, the side-wall layers, and finally other regions of intense shear. We consider both large- $S$ and small- $S$ cases.

\section{A.1. The core flow}

Using the equation for the vertical vorticity component, $\zeta=v_{x}-u_{y}$ of the vorticity vector, $\boldsymbol{\omega}=\nabla \times \mathbf{u}$, formed from (3.3b), and combining the vertical component of (3.3b) with $(3.3 c)$ leads to the equation pair

$$
\begin{gathered}
D \zeta-2 w_{z}-R o(\boldsymbol{\omega} \cdot \nabla) w=0, \\
\left(D^{2}+S\right) w=-D p_{z},
\end{gathered}
$$

where

$$
D \equiv \frac{\partial}{\partial t}+R o(\mathbf{u} \cdot \nabla)
$$

\section{A.1.1. Large $S$}

If $S \gg 1$, then from the second equation, $w=\mathcal{O}\left(S^{-1}\right)$, making the terms in (A 1) that involve $w$ negligible to leading order, so the equation reduces to

$$
D_{1} \zeta=0, \quad D_{1}=\frac{\partial}{\partial t}+R o\left(u \frac{\partial}{\partial x}+v \frac{\partial}{\partial y}\right) .
$$

So long as no pathlines enter the flow domain from the vertical walls - that is, that no eruption has yet occurred - we conclude that

$$
\zeta=2, \quad \text { for } t<t_{s} \text { and for all } z,
$$

where $t_{s}$ is the time for boundary-layer eruption into the core. The solution to this equation has been given by (2.3). Since the side walls are vertical, this equation indicates that $\mathbf{u}$ is $z$-independent; not only is the motion in horizontal planes, but it is also $z$ independent. So, then, since $p_{z}=0$, equation (A 2) indicates that $w$ is even smaller than $\mathcal{O}\left(S^{-1}\right)$.

Even after boundary-layer eruption, flows in regions away from singular wall layers and other regions of high shear are given by solutions to (A 4), but of course the solution is no longer (A 5).

\section{A.1.2. $S=\mathcal{O}(1)$}

The question is, of course, is the flow still two-dimensional? We know that vertical motion at the horizontal boundaries is of scale $E^{1 / 2}$, owing to eruption from the nonlinear Ekman layers on those surfaces. So, the only way in which larger vertical core motion can arise would be because of divergent horizontal motion, or from no-penetration at a sloping boundary. However, neither occurs here, so it appears that $w$ is sufficiently small to lead once again to $p_{z}=0$ and $D \zeta=0$ from (A 1), (A 2), and the motion is again two dimensional. So, the order of $S$ is apparently not relevant with regard to two-dimensionality of the core flow.

\section{A.2. The side-wall layer}

The side-wall boundary layer is two-dimensional in the small-Rossby-number case, as noted by (Foster \& Munro 2012), but it is less than obvious that is it two-dimensional for $R o=\mathcal{O}(1)$. Doing the usual boundary-layer approximations on the layer on $y=0+$, 
writing $y=E^{1 / 2} \bar{y}$ and $v=E^{1 / 2} \bar{v}$, the $x$ - and $y$-component equations are

$$
\begin{gathered}
u_{t}+\operatorname{Ro}\left(u u_{x}+\bar{v} u_{\bar{y}}+w u_{z}\right)-2 E^{1 / 2} \bar{v}+p_{x}=u_{\bar{y} \bar{y}} \\
2 E^{1 / 2} u+p_{\bar{y}}=0 .
\end{gathered}
$$

On integrating the continuity equation,

$$
E^{1 / 2} \bar{v}=\frac{1}{2} p_{x}-\frac{1}{2} Q_{x}(x, z, t)-E^{1 / 2} \int_{0}^{\bar{y}} w_{z}(x, Y, z, t) d Y .
$$

Putting $p_{x}$ from this expression into (A 6) leads to

$$
u_{t}+R o\left(u u_{x}+\bar{v} u_{\bar{y}}+w u_{z}\right)+Q_{x}(x, z, t)+2 E^{1 / 2} \int_{0}^{\bar{y}} w_{z}(x, Y, z, t) d Y=u_{\bar{y} \bar{y}},
$$

Clearly, to leading order, the integral term is negligible.

\section{A.2.1. Large $S$}

We know from $\S$ A.1.1 above that for $S \gg 1$, the interior motion is $z$-independent, so then the velocity at the layer's edge is $U(x, t)$, and hence $Q_{x}=-U_{t}-R o U U_{x}$. In that case, the first two terms in the pressure asymptotic series take the form

$$
p=\frac{1}{2} Q(x, t)+E^{1 / 2} p_{1}(x, \bar{y}, z, t)+\ldots .
$$

This result then leads to

$$
u_{\bar{t}}+u u_{x}+\bar{v} u_{\bar{y}}+w u_{z}+Q_{x}(x, z, \bar{t})=u_{\bar{y} \bar{y}}
$$

The equation for the vertical motion in the boundary layer is

$$
w_{t}+R o\left(u w_{x}+\bar{v} w_{\bar{y}}+w w_{z}\right)+E^{1 / 2} p_{1 z}=w_{\bar{y} \bar{y}}-\rho,
$$

and the density perturbation equation is

$$
\rho_{t}+R o\left(u \rho_{x}+\bar{v} \rho_{\bar{y}}+w \rho_{z}\right)-S w=0 .
$$

For $S=\mathcal{O}(1)$, it is evident from these equations that both $w$ and $\rho$ are order $E^{1 / 2}$. So, the $w$ convection term in (A 11) is negligible, leading to the two-dimensional boundary-layer equation (3.6), for which we have presented numerical solutions.

\section{A.2.2. $S=\mathcal{O}(1)$}

If the interior motion is two-dimensional and $z$-independent as suggested in $\S$ A.1.2, then equation (A 11) is again correct. Inspection of (A 12) and (A 13) shows that $w=\mathcal{O}\left(E^{1 / 2}\right)$ in this layer, and hence (A 11) again reduces to (3.6).

\section{A.3. Regions of high shear}

Intense vortices are evident in figures 4 and 5 , the result of erupted boundary-layer vorticity. When the Rossby number is small, it is well known that the cores of such vortices have width $E^{1 / 3}$, and in such regions, $w$ is of the same order as the swirl velocity there. Of course, when $R o$ exceeds $E^{1 / 2}$, that scaling is no longer correct. In this case, for $S=\mathcal{O}(1)$, the core thickness scales with $E^{1 / 2}$, and the vertical velocity in such a zone is of the same order as the swirl velocity, and hence the absence of particles in vortex cores of the latter images in figure 5 . For $S$ large, however, scale analysis of the vertical momentum equation indicates that the vortex core width is $(E S)^{1 / 2}$, so the core is thicker than in the smaller- $S$ case, as evident from the figures, and further the vertical 
velocity in the core scales with $S^{-1}$, so the particles are not swept out of the core in figure 4 . Hence, in the case of strong stratification, the vertical motion is inhibited, even in the vortex cores, but for $S=\mathcal{O}(1)$, significant vertical motion occurs.

\section{REFERENCES}

Benton, E. R. \& Clark, A. 1974 Spin-up. Annu. Rev. Fluid Mech. 6, 257-280.

Cebeci, T. 1986 Unsteady boundary layers with an intelligent numerical scheme. J. Fluid Mech. 163, $129-140$.

DAlziel, S. B. 2006 Digiflow user guide: http://www.damtp.cam.ac.uk/lab/digiflow/. .

Duck, P. W. \& Foster, M. R. 2001 Spin-up of homogeneous and stratified fluids. Annu. Rev. Fluid Mech. 33, 231-263.

Duck, P. W., Foster, M. R. \& Hewitt, R. E. 1997 On the boundary layer arising in the spin-up of a stratified fluid in a container with sloping walls. J. Fluid Mech. 335, 233-259.

Economidou, M. \& Hunt, G. R. 2009 Density stratified environments: the double-tank method. Exp. Fluids 46, 453-466.

Foster, M. R. \& Munro, R. J. 2012 The linear spin-up of a stratified, rotating fluid in a square cylinder. J. Fluid Mech. 712, 7-40.

Greenspan, H. P. \& Howard, L. N. 1963 On a time-dependent motion of a rotating fluid. J. Fluid Mech. 17, 385-404.

Heil, M. \& Hazel, A.L. 2006 oomph-lib-An Object-Oriented Multi-Physics Finite-Element Library. In Fluid-Structure Interaction (ed. M. Schafer \& H.-J. Bungartz), p. 19. Springer (Lecture notes on computational science and engineering).

Hewitt, R. E., Davies, P. A., Duck, P. W. \& Foster, M. R. 1999 Spin-up of stratified rotating flows at large schmidt number: experiment and theory. J. Fluid Mech. 389, 169207.

Hewitt, R. E., Foster, M. R. \& Davies, P. A. 2001 Spin-up of a two-layer rotating stratified fluid in a variable-depth container. J. Fluid Mech. 438, 379-407.

Hewitt, R. E., Hazel, A. L., Clarke, R. J. \& Denier, J. P. 2011 Unsteady flow in a rotating torus after a sudden change in rotation rate. J. Fluid Mech. 688, 88-119.

Koumoutsakos, P. \& Leonard, A. 1995 High-resolution simulations of the flow around an impulsively started cylinder using vortex methods. J. Fluid Mech. 296, 1-38.

MacCready, P. \& Rhines, P. B. 1991 Buoyant inhibition of ekman transport on a slope and its effect on stratified spin-up. J. Fluid Mech. 223, 631-661.

Munro, R. J., Foster, M. R. \& Davies, P. A. 2010 Instabilities in the spin-up of a rotating, stratified fluid. Phys. Fluids 22(5), 054108.

Smirnov, S. A., Baines, P. G., Boyer, D. L., Voropayev, S. I. \& Srdic-Mitrovic, A. N. 2005 Long-time evolution of linearly stratified spin-up flows in axisymmetric geometries. Phys. Fluids 17 (1), 016601.

Stewartson, K. 1957 On almost rigid rotations. J. Fluid Mech. 3, 17-26.

Thomas, L. N. \& Rhines, P. B. 2002 Nonlinear stratified spin-up. J. Fluid Mech. 473, 211-244.

van de Konijnenberg, J. A. \& van Heijst, G. J. F. 1997 Free-surface effects on spin-up in a rectangular tank. J. Fluid Mech. 334, 189-210.

VAN HeIJst, G. J. F. 1989 Spin-up phenomena in non-axisymmetric containers. J. Fluid Mech. 206, 171-191.

van Heijst, G. J. F., Davies, P. A. \& Davis, R. G 1990 Spin-up in a rectangular container. Phys. Fluids A 2, 150-159.

WAlin, G. 1969 Some aspects of time-dependent motion of a stratified rotating fluid. J. Fluid Mech. 36(2), 289-307.

Weidman, P. D. 1976 On the spin-up and spin-down of a rotating fluid. Part 1. Extending the Wedemeyer model. J. Fluid Mech. 77(4), 685-708. 